Check for updates

Cite this: J. Mater. Chem. B, 2021 9, 6973

Received 14th April 2021, Accepted 13th May 2021

DOI: $10.1039 / \mathrm{d} 1 \mathrm{tb} 00843 a$

rsc.li/materials-b

\section{Bringing naturally-occurring saturated fatty acids into biomedical research $\dagger$}

\begin{abstract}
Ke Xue, $\$$ Shuyi Lv $\ddagger$ and Chunlei Zhu (D) *
Naturally-occurring saturated fatty acids (NSFAs) have emerged as a class of promising biomaterials due to their low cost, chemical stability, well-defined melting points, large heat of fusion, reversible solidliquid phase transition, biocompatibility, biodegradability, and inherent antibacterial activity. By virtue of these unique advantages, a plethora of attempts have been made by taking NSFAs as gating materials for controlled release or simply serving as bioactive substances for the manipulation of bacterial/cellular behaviors, which greatly boosts their widespread applications in biomedical research. In this review, we systematically summarize the advances of NSFA-based materials in the biomedical field over the past decade. We begin with an introduction to NSFAs and their physiochemical/biological properties, with an emphasis on the working mechanism for controlled release. We then discuss current approaches for the fabrication of colloidally dispersed NSFA-based materials. Further, we showcase the specific applications of NSFA-based materials in biomedical research, including controlled drug release, targeted drug delivery, cancer therapy, antibacterial treatment, and tissue engineering. Lastly, this review is concluded with a summary and perspectives on future directions.
\end{abstract}

\section{Introduction}

Naturally-occurring saturated fatty acids (NSFAs) are straightchain carboxylic acids with an even number of carbon atoms. ${ }^{1-3}$ A variety of living organisms ranging from plants to animals are able to produce NSFAs, which, in return, serve as important

Key Laboratory of Functional Polymer Materials of Ministry of Education, State Key Laboratory of Medicinal Chemical Biology, Institute of Polymer Chemistry, College of Chemistry, Nankai University, Tianjin, 300071, China. E-mail: chunlei.zhu@nankai.edu.cn

$\dagger$ Dedicated to the 100th anniversary of Chemistry at Nankai University.

$\ddagger$ These authors contributed equally to this work. structural components and dietary energy sources to facilitate cell proliferation, cell metabolism, and/or signal transduction. ${ }^{4-7}$ In view of the large accessibility as well as the functional diversity, NSFAs have found widespread applications in our daily life, such as acting as surfactants, food additives, and plasticizers in the cosmetics and food industries and serving as thermo-sensitive materials in the building and construction areas. ${ }^{8-11}$

In terms of heat management with NSFAs, both the phasetransition temperature and the latent heat of fusion determine the actual effect of temperature control. As a matter of fact, NSFAs are a subset of phase-change materials (PCMs), which are typically defined as a certain class of substances featuring

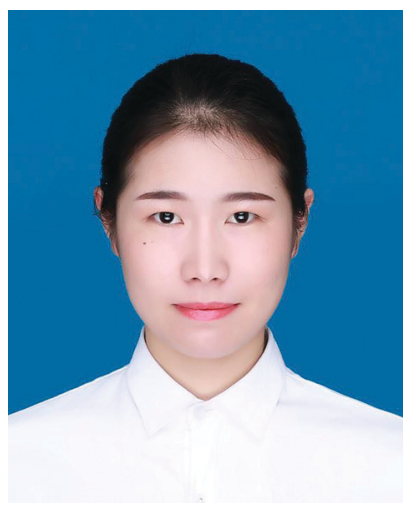

Ke Xue
Ke Xue received her $B S$ from Qingdao University in 2015 and MS from Shandong Normal University in 2018. She is currently pursuing her PhD degree in Polymer Chemistry and Physics at Nankai University under the supervision of Prof. Chunlei Zhu. Her research interest focuses on the construction of saturated fatty acidbased systems for biomedical applications.

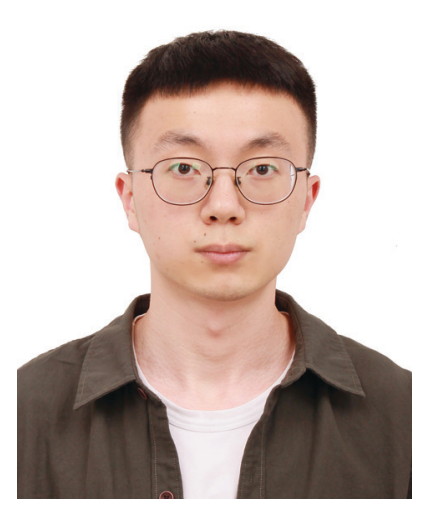

Shuyi Lv received his BS in Polymer Materials and Engineering from Yunnan University in 2019. He is currently pursuing his PhD degree in Polymer Chemistry and Physics at Nankai University under the supervision of Prof. Chunlei Zhu. His is now working on the design and synthesis of functional fluorescent probes for bacterial labeling.
Shuyi Lv 
Table 1 The classification and properties of PCMs

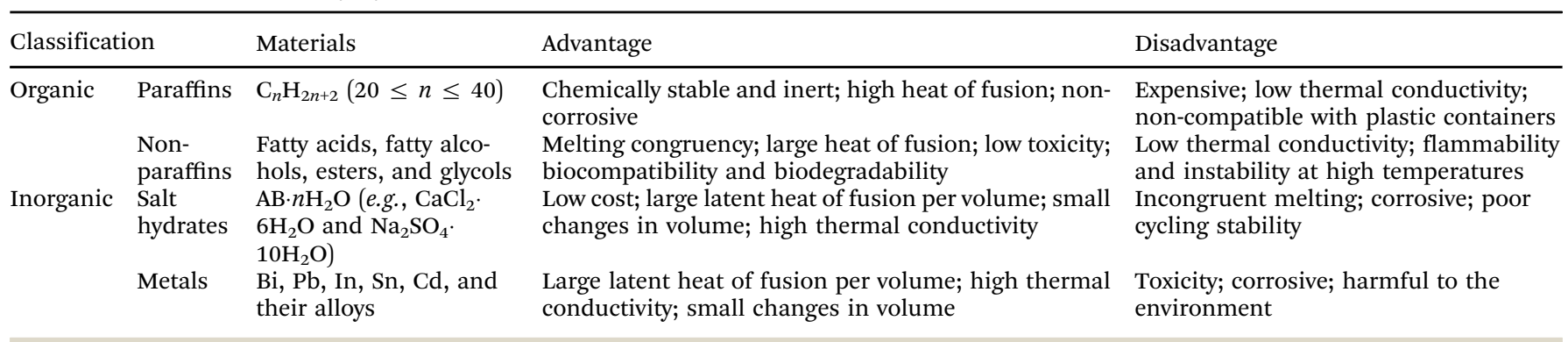

large latent heat during a phase-transition process. ${ }^{12,13}$ Among the three types of phase transitions (i.e., solid-solid, solidliquid, and liquid-gas), the solid-liquid transition is most commonly used due to the low transition temperature, small volume change, high latent energy, and good thermal conductivity. According to the chemical composition, solid-liquid PCMs can be broadly divided into two major categories, that is, inorganic and organic PCMs. ${ }^{13-15}$ Notable examples include salt hydrates and metals (for inorganic PCMs) as well as paraffins and non-paraffins (for organic PCMs) (Table 1). In spite of the advantages of high thermal conductivity, high heat of fusion per volume, and small volume change, inorganic PCMs also exhibit some evident drawbacks, such as poor cycling stability, high toxicity, and corrosiveness, limiting their applications in biological systems. ${ }^{15-17}$ In contrast, organic PCMs have the virtue of large latent heat of fusion, noncorrosiveness, and low toxicity. Owing to the high price of pure paraffins that are characterized by the chemical formula of $\mathrm{C}_{n} \mathrm{H}_{2 n+2}(20 \leq n \leq 40)$, non-paraffin PCMs (e.g., fatty acids, fatty alcohols, esters, and glycols) have emerged as a class of practical materials for real-world applications. ${ }^{18,19}$ Among various non-paraffin PCMs, bio-based NSFAs have attracted extensive attention. In addition to the general advantages for organic PCMs, NSFAs also possess low cost, little or no supercooling during

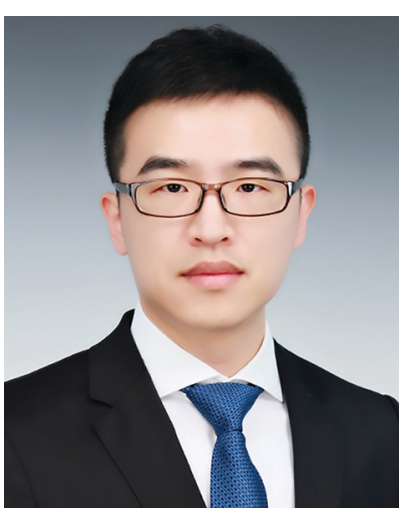

Chunlei Zhu
Chunlei $Z$ hu received his $B S$ from Jilin University in 2008 and $P h D$ from the Institute of Chemistry, Chinese Academy of Sciences in 2013 with Prof. Shu Wang. He then conducted his postdoctoral research with Prof. Younan Xia at the Georgia Institute of Technology from 2014 to 2017 and Prof. Ben Zhong Tang at the Hong Kong University of Science and Technology from 2017 to 2018. He started his independent academic career in the College of Chemistry at Nankai University in 2018. His current research interest includes the design and fabrication of functional organic and/or polymeric materials for biomedical applications. the phase transition, and, most importantly, biocompatibility and biodegradability, enabling them to be a class of attractive biomaterials. ${ }^{11,13,20,21}$ Taking advantage of the reversible solidliquid phase transition, NSFAs can function as gating materials to achieve controlled release, which remarkably extends the applications of NSFAs and opens a multitude of new avenues in biomedical research. ${ }^{13,22-24}$ It should be noted that, in contrast to NSFAs, unsaturated fatty acids that feature one or more double bonds are chemically less stable, and are susceptible to oxidation. In addition, due to the weak intermolecular interactions, unsaturated fatty acids typically exist in the liquid form at room temperature (e.g., oleic acid, m. p. $=13.4{ }^{\circ} \mathrm{C}$ ), making them not suitable for firm drug encapsulation and on-demand drug release in vivo.

In this review, we systematically summarize the advances of NSFAs in the biomedical field over the past decade. We begin with an introduction to NSFAs and their physiochemical/biological properties, in which the unique advantages of NSFAs in biomedicine as well as their working mechanism for controlled release are discussed. To facilitate the biomedical applications of NSFAs, it is essential to establish robust strategies to formulate these highly crystalline materials into colloidally stable particles. In this regard, we summarize current approaches for the fabrication of colloidally dispersed NSFA-based materials. We then showcase the specific applications of NSFAbased materials in biomedical research, including controlled release, targeted drug delivery, cancer therapy, antibacterial treatment, and tissue engineering (Fig. 1). Lastly, we conclude this review with a summary and perspectives on future directions.

\section{Structure and properties of NSFAs}

\subsection{Chemical structure and physiochemical/biological properties}

NSFAs are typically referred to as straight-chain organic acids with the general structure formula $\mathrm{C}_{n} \mathrm{H}_{2 n} \mathrm{O}_{2}(n \geq 10)$, which are derived from animal fats and/or plant oils. ${ }^{25}$ Structurally, each type of NSFAs contains a carboxyl group located at one end of the molecule and a linear saturated alkyl chain composed of an even number of carbon atoms. As the number of carbon atoms increases from 10 to 18 , the corresponding NSFAs are also known as capric acid (m. p. $\left.=31.6{ }^{\circ} \mathrm{C}, \mathrm{CA}\right)$, lauric acid $(\mathrm{m} . \mathrm{p} .=$ $43.8{ }^{\circ} \mathrm{C}, \mathrm{LA}$ ), myristic acid (m. p. $=54.4{ }^{\circ} \mathrm{C}, \mathrm{MA}$ ), palmitic acid (m. p. $\left.=62.9{ }^{\circ} \mathrm{C}, \mathrm{PA}\right)$, and stearic acid (m. p. $\left.=69.3{ }^{\circ} \mathrm{C}, \mathrm{SA}\right)$, 


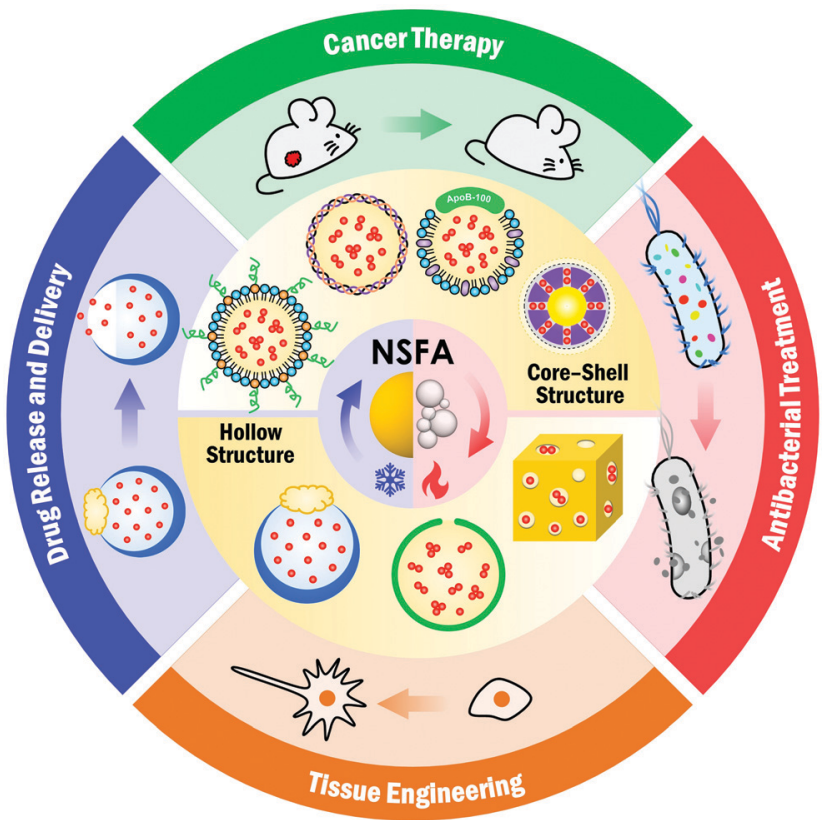

Fig. 1 An overview of NSFA-based materials and their applications in biomedical research.

respectively. ${ }^{11,13,26}$ Since the melting point of CA is higher than $25{ }^{\circ} \mathrm{C}$, all commonly used NSFAs exist in the solid form at room temperature. In principle, the increase in the length of the alkyl chains leads to a variety of changes regarding the physiochemical properties of NSFAs due to the strengthened intermolecular interactions and/or molecular packing, such as increased melting points, decreased water solubility, and enhanced crystallinity. ${ }^{11,22}$ Biologically, NSFAs are essential nutrients and energy sources for organisms, which can be obtained by either extracellular ingestion or intracellular de novo synthesis. ${ }^{4,5}$ The utilization of NSFAs involves a metabolic process called $\beta$-oxidation, in which long fatty acids are successively broken down into acetoacetyl coenzyme A (acetyl-CoA) that subsequently enters another metabolic process called the Krebs cycle to produce energy (i.e., ATP). ${ }^{27-29}$ In spite of the pivotal role of NSFAs for organisms, the intake of excessive free NSFAs by cells is considered to be detrimental, which is presumably ascribed to the detergent-like behaviors of NSFAs to disrupt membranes and proteins. ${ }^{30,31}$ To mitigate the lipotoxic effects and the elevated oxidative stress, NSFAs are typically esterified in the endoplasmic reticulum and then stored in the form of triglycerides, phospholipids, and cholesterol esters, which act as important dietary energy supplies and structural components of cells. ${ }^{32,33}$

\subsection{Working mechanism for controlled release}

It is universally acknowledged that PCMs can undergo reversible phase transitions in response to heat stimulation, rendering them a class of promising thermo-responsive materials for controlled release (Fig. 2). ${ }^{23,24}$ In the solid state, NSFA molecules tend to closely pack with each other due to the strong intermolecular interactions (e.g., hydrogen bonding, van der Waals forces, and hydrophobic stacking), resulting in high crystallinity and low water solubility. ${ }^{13,22}$ In this case, the payloads are firmly locked within the NSFA matrix, preferably in the form of self-aggregates. ${ }^{34,35}$ In the presence of internal and/or external stimuli (e.g., direct heating, near-infrared (NIR) light, ultrasound, and magnetic fields), NSFAs absorb heat to reach the melting point, and the type of energy stored by NSFAs starts to change from sensible heat (related to temperature changes) to latent heat (related to phase changes). ${ }^{23,24,36}$ During this process, NSFAs are subjected to a solid-liquid phase transition and are eventually melted into transparent liquid. In this situation, the intermolecular forces of the NSFAs become weakened, resulting in drastic variations in a set of physicochemical properties, including increased molecular

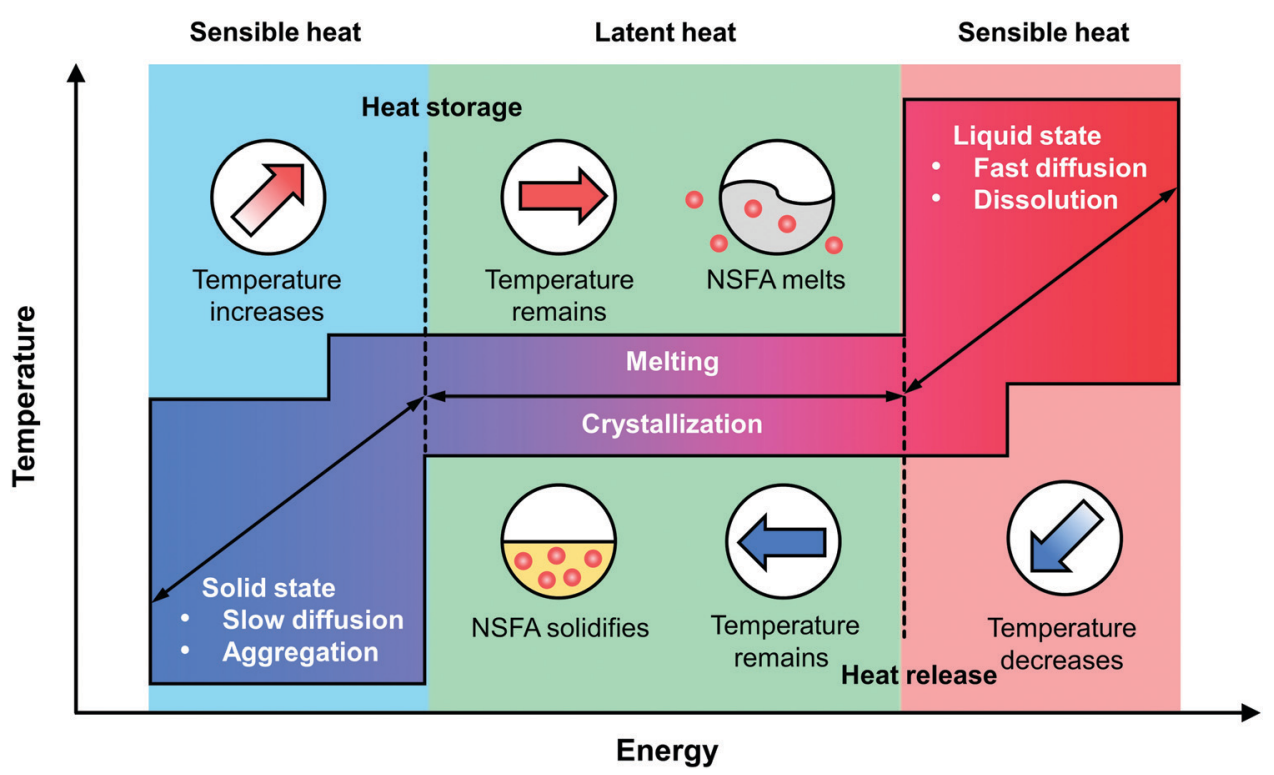

Fig. 2 Working mechanism of NSFAs for controlled release. 
mobility, reduced viscosity, and decreased density. ${ }^{13,22}$ These changes significantly favor the fast dissolution and efficient diffusion of payloads that are initially trapped in the solid matrix, thereby giving rise to swift release. Since the phase transition is highly reversible, liquid NFSAs can also experience a reverse process to recover to the original solid state via crystallization by releasing sensible and latent heat, making the unreleased payloads frozen again in the NFSA matrix. ${ }^{11,13,22}$ Accordingly, by manipulating the phase-change process of NSFAs with strategies that are able to cause temperature variations, it is possible to achieve on-demand drug release.

\subsection{Eutectic mixtures of NSFAs}

Despite the fact that the melting points of single-component NSFAs (carbon number $=10-18$ ) are available from 31.6 to $69.3{ }^{\circ} \mathrm{C}$, there is still a lack of an NSFA-based system with a phase-transition temperature slightly beyond the physiological temperature of human bodies (i.e., $37{ }^{\circ} \mathrm{C}$ ) to ensure the close packaging of payloads during blood circulation and thus reduce off-target toxicity. In addition, single-component NSFAs tend to crystallize during solidification, which inevitably results in the exclusion of payloads from the NSFA matrix and thus generates a drug-rich outer layer for undesired burst release. ${ }^{35,37-39}$ In this regard, a eutectic mixture of NSFAs can provide an alternative way to address the aforementioned shortcomings. A eutectic mixture typically refers to a mixture of two or more components that do not interact to form a new homogenous entity; under a particular ratio, the mixture melts or solidifies at a temperature that is lower than the melting point of either of its components. ${ }^{11,40}$ The capacity of NSFAs to form binary or ternary eutectics greatly expands the available melting points (Table 2), and, meanwhile, alleviates the high crystallinity of single-component NSFAs for improved drug loading. ${ }^{41-49}$ For instance, Zhu et al. reported a eutectic mixture of LA and SA that were doped at a weight ratio of $4: 1{ }^{24}$ Under such circumstances, the mixture exhibited a well-defined melting point of $39{ }^{\circ} \mathrm{C}$, which was highly preferred for practical use due to its proximity to physiological temperature. Furthermore, they formulated these materials into NIR-responsive, drug-loaded nanoparticles and achieved controlled drug release for the killing of cancer cells.

\section{Strategies for the fabrication of stable NSFA-based materials}

To facilitate the biomedical applications of NSFAs, it is necessary to formulate them into stable particles. Despite the fact that a plethora of methods have been established for the conversion of hydrophobic materials into colloidally stable nanoparticles, few of them can be directly borrowed to fabricate NSFA-based materials due to their high crystallinity. ${ }^{13,22,35}$ Herein, we summarize currently developed approaches for the transformation of NSFAs into stable particles, which can be broadly classified into two major categories, that is, the formation core-shell and pore structured particles.
Table 2 The proportion and melting points of various eutectic mixtures of NSFAs

\begin{tabular}{|c|c|c|c|}
\hline Eutectics & Weight ratio & Melting point $\left({ }^{\circ} \mathrm{C}\right)$ & Ref. \\
\hline \multirow[t]{3}{*}{ CA:LA } & $61.5: 38.5$ & 19.1 & 41 \\
\hline & $64: 36$ & 19.6 & 47 \\
\hline & $67: 33$ & 22.8 & 42 \\
\hline \multirow[t]{2}{*}{$\mathrm{CA}: \mathrm{MA}$} & $74: 26$ & 22.2 & 48 \\
\hline & $72: 28$ & 25.4 & 42 \\
\hline \multirow[t]{2}{*}{ CA: PA } & $76.5: 23.5$ & 21.9 & 43 \\
\hline & $75.2: 24.8$ & 22.1 & 41 \\
\hline \multirow[t]{3}{*}{ CA: SA } & $83: 17$ & 25.4 & 47 \\
\hline & $86.6: 13.4$ & 26.8 & 41 \\
\hline & $77: 23$ & 27.8 & 42 \\
\hline \multirow[t]{3}{*}{ LA : MA } & $58: 42$ & 35.2 & 42 \\
\hline & $61.3: 38.7$ & 33.3 & 46 \\
\hline & $66: 34$ & 34.2 & 45 \\
\hline \multirow{2}{*}{ LA: PA } & $69: 31$ & 35.2 & 41 \\
\hline & $77.5: 22.5$ & 33.6 & 46 \\
\hline \multirow[t]{2}{*}{ LA: SA } & $75.5: 24.5$ & 36.7 & 41 \\
\hline & $80: 20$ & 39.0 & 24 \\
\hline \multirow[t]{2}{*}{ MA : PA } & $58: 42$ & 42.6 & 44 \\
\hline & $66.9: 33.1$ & 45.4 & 46 \\
\hline \multirow[t]{2}{*}{ MA: SA } & $64: 36$ & 44.1 & 45 \\
\hline & $77.4: 22.6$ & 46.4 & 46 \\
\hline \multirow[t]{2}{*}{ PA:SA } & $63: 37$ & 53.7 & 46 \\
\hline & $64.2: 35.8$ & 53.2 & 41 \\
\hline LA: MA:SA & $57.5: 34.3: 8.2$ & 29.5 & 49 \\
\hline MA: SA: PA & $52.5: 17.2: 30.3$ & 40.9 & 49 \\
\hline
\end{tabular}

\subsection{Core-shell structure}

Core-shell structured particles are characterized by an inner core that primarily maintains the fundamental properties of the processed materials, and an outer shell that functions as a coating material to provide structural stability and integrity. ${ }^{50,51}$ The formation of core-shell structures is an effective strategy to transform bulk NSFAs into stable particles, which can be accomplished by three types of fabrication approaches (Fig. 3). The first one is to create the hydrophobic core that is made of NSFAs and payloads, followed by the association and/or in situ production of the hydrophilic shell. The second one is to prepare the hollow shell structures in advance, followed by the loading of NSFAs and payloads via physical diffusion. The third one is to employ NSFAs as a protective shell to seal existing drug-loaded particles with surface openings.

3.1.1. Stabilizing NSFAs with amphiphiles. Amphiphiles are classical coating materials for the stabilization of nanoparticles. In principle, amphiphiles consist of a hydrophilic head (either ionized or non-ionized) and a hydrophobic tail; in aqueous solution, these amphiphilic molecules tend to self-assemble into a variety of supramolecular structures to minimize the free energy. ${ }^{52-54}$ In this scenario, the hydrophilic portion extends to the aqueous phase, while the hydrophobic portion intercalates into the core of NSFAs. Notable amphiphiles include natural phospholipids, synthetic phospholipids, and poloxamers (e.g., Pluronic ${ }^{\circledR}$ F127). ${ }^{24,39,55}$ In an example, Zhu et al. reported the use of 1,2-distearoyl-sn-glycero-3-phosphoethanolamine $N$-[methoxy(poly(ethylene glycol))-5000] (DSPE-PEG) and lecithin for the fabrication of stable NSFA nanoparticles (Fig. 3A). ${ }^{24}$ Because of the universality of this approach, a variety of payloads with appropriate hydrophobicity have been 

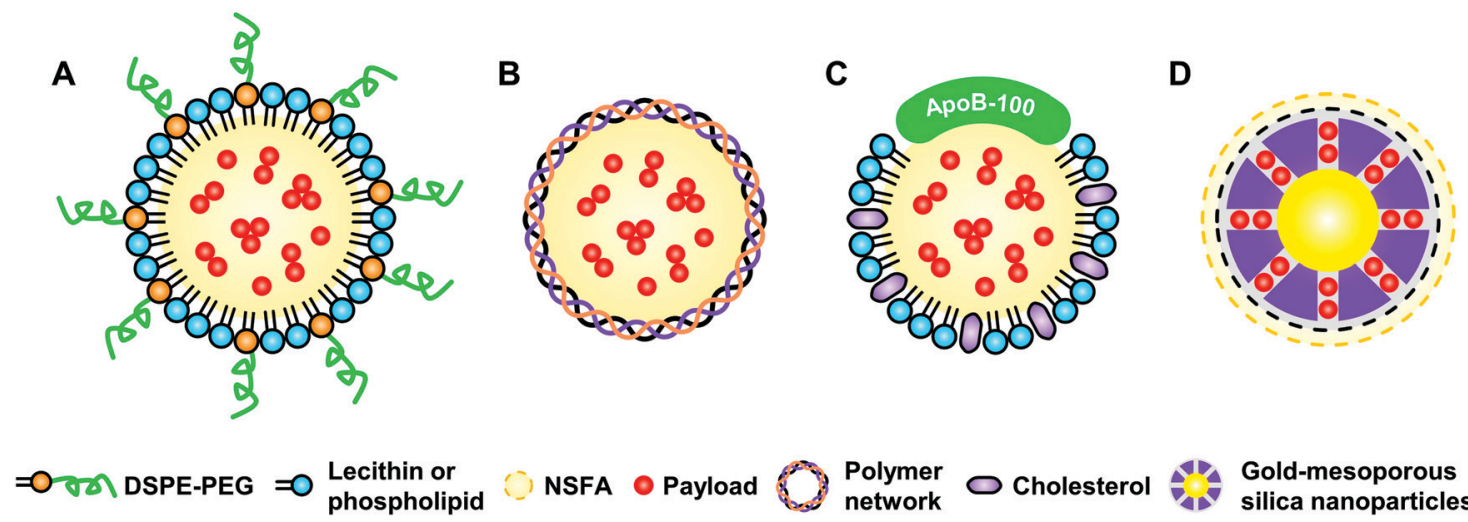

NSFA Payload

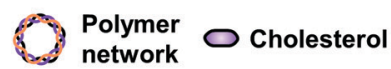

Gold-mesoporous silica nanoparticles

Fig. 3 Core-shell structured NSFA-based particles. (A) Stabilizing with amphiphiles. (B) Stabilizing via in situ surface polymerization. (C) Stabilizing with native biological templates. (D) Sealing drug-loaded particles with NSFAs.

successfully encapsulated into NSFA nanoparticles, such as anticancer drugs, antibiotics, and/or NIR dyes. ${ }^{24,56}$ Besides, based on the established synthetic methods for the modification of DSPE-PEG molecules, it is possible to endow NSFA nanoparticles with additional functionalities, such as tumortargeting capability by conjugation with specific ligands (e.g., folic acid). ${ }^{55,57}$ It is noteworthy that DSPE-PEG, lecithin, and NSFAs are all safe for clinical use, indicating the potential of this system for real-world applications.

3.1.2. Stabilizing NSFAs via in situ surface polymerization. In addition to direct coating of NSFA particles with amphiphilic molecules, in situ surface polymerization has been demonstrated as another effective strategy for the stabilization of NSFAs. ${ }^{58}$ In this technique, an emulsion of NSFAs is first prepared in aqueous solution, followed by the addition of appropriate monomers, cross-linkers, and initiators for in situ polymerization to proceed on the surface of NSFA particles, which provides good colloidal stability through the formation of a hydrophilic polymer network. In principle, the density of the resulting polymer network can be adjusted simply by varying the concentration of monomers and/or cross-linkers, the polymerization time, and the reaction temperature; meanwhile, the surface properties can be readily tuned by using monomers with appropriate properties, such as ionic ones (e.g., cationic, anionic, and zwitterionic) or the ones with chemically modifiable groups (e.g., $\mathrm{NH}_{2}$ and $\left.\mathrm{COOH}\right) .{ }^{5-61}$ Different from amphiphiles with long alkyl chains that presumably interact with NSFAs and impair their physicochemical properties, in situ surface polymerization only generates a polymer network outside the inner core, which maximally maintains the performance of NSFAs. In a recent study, our group demonstrated the possibility of fabricating colloidally stable NSFA nanoparticles using this technique (Fig. 3B).$^{58}$ In particular, a positively charged monomer was employed to tune the surface charge of the resulting nanoparticles, which was helpful to enhance their electrostatic interactions with negatively charged bacteria.

3.1.3. Stabilizing NSFAs with native biological templates. In recent years, native biological materials have been widely exploited as novel nanocarriers for drug delivery, such as exosomes, lipoproteins, and viruses. ${ }^{62-64}$ Among them, low-density lipoprotein (LDL) particles have received considerable attention due to their small size $(<50 \mathrm{~nm})$, excellent biocompatibility and biodegradability, natural tumor-targeting capability, and long blood circulation time (2-4 days). ${ }^{6-67}$ Structurally, each LDL consists of a monolayer of amphiphilic phospholipids, one apolipoprotein B-100 (ApoB-100), and a hydrophobic core primarily made of endogenous triglycerides and cholesterol esters. By means of the "like dissolves like" principle, the core lipids can be efficiently removed by nonpolar organic solvents (e.g., hexane and toluene), leaving a large hydrophobic space for the accommodation of exogenous materials with appropriate hydrophobicity (e.g., contrast agents and photosensitizers). ${ }^{67,68}$ In view of these prominent advantages, Zhu et al. reported the core-reconstitution of LDL particles with a eutectic mixture of NSFAs and an anticancer drug (Fig. 3C). ${ }^{67}$ Since the surface intercalated ApoB-100 could specifically bind with the overexpressed LDL receptors in malignant cells, the reconstituted LDL particles showed targeted cytotoxicity toward cancer cells over healthy cells.

3.1.4. Sealing drug-loaded particles with NSFAs. Although NSFAs are mostly employed as the core materials, they can also serve as the shell materials to prevent payload leakage from porous particles before arriving at the target site. ${ }^{69-72}$ In a typical process, the void space in porous particles (e.g., mesoporous silica nanoparticles) is first filled with appropriate payloads, followed by sealing with a solid NSFA shell as the gatekeeper for stimuli-responsive release (Fig. 3D). ${ }^{70-72}$ In an example, Zhou and coworkers used a eutectic mixture of LA and SA to block urokinase-loaded gold-mesoporous silica coreshell nanoparticles. ${ }^{72}$ Taking advantage of the photothermal effect of gold nanoparticles, they further achieved NIR-triggered release of urokinase for the treatment of deep vein thrombosis. In another study, Dong and coworkers reported a multifunctional nanoparticle that consisted of manganese silicate-supported calcium peroxide ( $\left.\mathrm{MSN} @ \mathrm{CaO}_{2}\right)$, indocyanine green (ICG), and a layer of LA. ${ }^{73}$ Upon NIR irradiation, ICG boosted the generation of reactive oxygen species (ROS) and heat to trigger the melting of LA, followed by the exposed $\mathrm{CaO}_{2}$ reacting with water to produce $\mathrm{H}_{2} \mathrm{O}_{2}$ and $\mathrm{O}_{2}$ for hypoxia-relieved photodynamic therapy (PDT). In addition, the interaction between MSNs and glutathione 
led to the release of Fenton-like catalyst $\mathrm{Mn}^{2+}$ for $\mathrm{H}_{2} \mathrm{O}_{2}$ supplied, magnetic resonance imaging (MRI)-guided chemodynamic therapy (CDT).

\subsection{Hollow structure}

Since NSFAs are difficult to transform into stable particles without the addition of appropriate stabilizers, hollow materials with one or more accessible pores can be utilized as the supporting framework for drug loading, followed by plugging with solid NSFAs or simultaneous filling with NSFAs and payloads. Upon certain stimuli to trigger the melting of NSFAs, the encapsulated payloads are rapidly released to perform specific chemical and/or biological functions.

3.2.1. Single-hole particles. To facilitate efficient release of payloads, it is preferable to build a hollow particle with a single hole on the shell surface to serve as the container of hydrophilic molecules in an aqueous solution. ${ }^{74-77}$ In a following step, the single-hole particles are sealed by solid NSFAs to form a bottlecork structure, thereby preventing the undesired leakage of payloads (Fig. 4A). ${ }^{77-79}$ The corked NSFAs are subjected to a solid-liquid transition when the temperature is raised beyond the melting point, resulting in the quick release of the sealed payloads. Xia and coworkers demonstrated this concept by fabricating hollow polystyrene particles featuring an open hole on the surface. ${ }^{78}$ After filling the voids with aqueous Rhodamine B solution, these particles were sealed with a cork made of a mixture of LA and 1-tetradecanol to achieve temperaturecontrolled drug release. In a recent study, they reported another type of single-hole silica particles, which were obtained by using gold-polystyrene Janus nanoparticles as the template, followed by silica deposition on the surface of polystyrene and subsequent template removal (Fig. 4B). ${ }^{79}$ Furthermore, they loaded a eutectic mixture of LA and SA together with an NIR dye (ICG) and an anticancer drug into the resulting silica nanocapsules for NIR-triggered drug release.

3.2.2. Multi-hole particles. To date, a variety of multi-hole particles have been developed to facilitate the loading of NSFAs for controlled drug delivery, including gold nanocages, mesoporous silica, 3D porous carbon materials, metal-organic frameworks, and hollow bismuth selenide nanoparticles. ${ }^{36,70-72,80,81}$ In contrast to single-hole particles, multi-hole particles can provide more pathways for NSFAs and payloads to enter the internal cavities. To improve the loading efficiency, both degassing in a vacuum and solvent-assisted diffusion have been employed to maximally occupy the voids of hollow particles with NSFAs and payloads. ${ }^{22,36}$ In a pioneering study, Xia and coworkers reported the fabrication of gold nanocages through a galvanic replacement reaction between silver nanocubes and $\mathrm{HAuCl}_{4}{ }^{82,83}$ Owing to the unique property of surface plasmon resonance, gold nanocages exhibit strong absorption in the NIR region, rendering them prominent photothermal agents. ${ }^{84}$ In view of the multi-hole structure, inherent photothermal effect, and chemical inertness, gold nanocages have been widely explored for the encapsulation of NSFAs and payloads. ${ }^{36,85-89}$ In a typical process, a mixture of NSFAs and payloads is diffused into gold nanocages through the holes at a
A

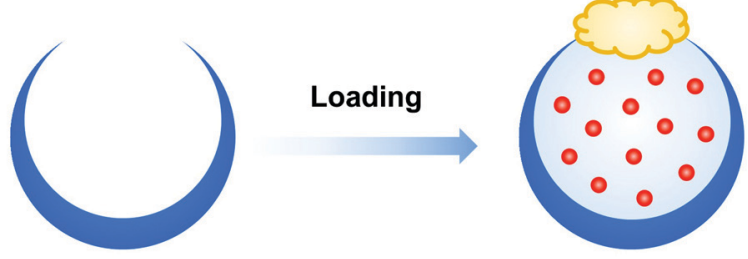

B

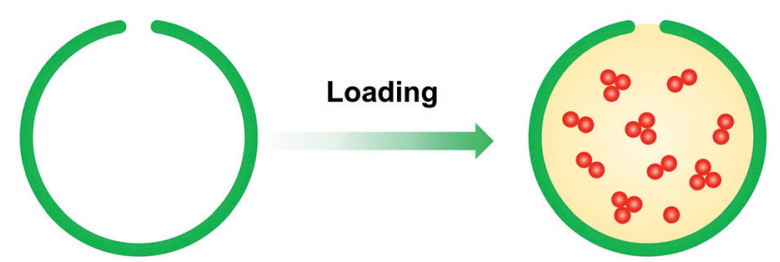

C

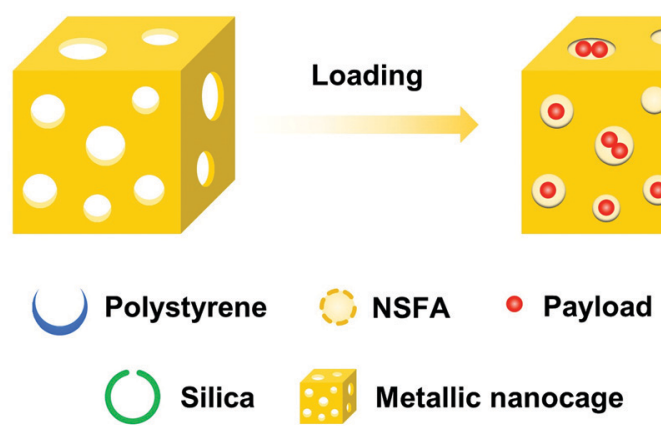

Fig. 4 Pore structured NSFA-based particles. (A) A single-hole polystyrene particle capped with NSFAs. (B) A single-hole silica nanoparticle filled with NSFAs. (C) A metallic nanocage filled with NFSAs.

temperature higher than the melting point of the NSFAs. When the samples are cooled down to room temperature, the NSFAs become solidified in the cavities of the gold nanocages to achieve efficient drug loading (Fig. 4C). Upon NIR irradiation, the loaded NSFAs undergo a solid-liquid phase transition due to the photothermal effect of the gold nanocages, which triggers the swift release of the encapsulated payloads. ${ }^{36,86-89}$

\section{Biomedical applications of NSFAs}

By virtue of the unique features in terms of low cost, chemical stability, well-defined and versatile melting points, large heat of fusion, reversible solid-liquid phase transition, biocompatibility, biodegradability, and inherent antibacterial activity, NSFAs have found a wide spectrum of biomedical applications using the aforementioned stabilization strategies, including controlled drug release, targeted drug delivery, cancer therapy, antibacterial treatment, and tissue engineering. These applications are separately discussed in the following sections.

\subsection{Controlled release}

For in vivo drug delivery, nanoparticles need to experience many steps or pass through a series of biological barriers to reach the diseased site (e.g., blood circulation and the bloodbrain barrier). During this process, off-target drug release 
should be strictly restricted as it may cause undesired toxicity to normal cells and healthy tissues as well as decreased drug accumulation in the diseased site. As such, stimuli-responsive drug delivery systems are particularly attractive to achieve on-demand drug release. ${ }^{90-92}$ To date, a variety of external and internal stimuli have been employed to control the release behaviors of payloads, including light, magnetic fields, ultrasound, temperature, $\mathrm{pH}$, redox, and enzymes. ${ }^{23,24,36,93-95}$ As a novel class of thermo-responsive materials, NSFAs are able to exhibit rapid, reversible solid-liquid transitions in response to temperature variations, which occur at all times in human beings as the local body temperature dynamically fluctuates depending on ambient conditions and pathological changes (e.g., inflammation, virus infection, and the tumor microenvironment). ${ }^{13,22,96,97}$ In this regard, NSFAs can be utilized as gating materials to control the release profiles of payloads. In 2010, Xia and coworkers demonstrated this concept by constructing a dual temperature-controlled system for drug release, in which 1-tetradecanol (another type of PCMs, m. p. = 38-39 $\left.{ }^{\circ} \mathrm{C}\right)$ and LA (m. p. $\left.=44{ }^{\circ} \mathrm{C}\right)$ served as the gatekeepers to regulate the release behaviors of fluorescein isothiocyanatedextran (FITC-dextran) from uniform gelatin microbeads (Fig. 5A). ${ }^{23}$ Due to the difference in the melting points between 1-tetradecanol and LA, FITC-dextran exhibited a two-stage release profile at 39 and $44{ }^{\circ} \mathrm{C}$ from gelatin microbeads that were pre-embedded in block matrices of 1-tetradecanol and LA, respectively, with basically no release at $37{ }^{\circ} \mathrm{C}$.

Although direct heating is the most straightforward way to trigger solid-liquid transitions of NSFAs, it cannot be applied to a specific subcutaneous area. Given the noninvasiveness, deep penetration in soft tissues, and high spatiotemporal resolution, NIR light can be employed as an external stimulus to locally induce temperature increase via efficient photothermal conversion when the system is doped with photothermal agents, such as gold nanocages, iron oxide nanoparticles, graphene, black phosphorus, copper-based nanomaterials, and organic NIR dyes. ${ }^{58,98-101}$ In a typical example, Zhu et al. fabricated an NIR-responsive, phospholipid-stabilized NSFA nanoparticle, whose inner core was composed of a eutectic mixture of LA and SA with a melting point of $39{ }^{\circ} \mathrm{C}$, an anticancer drug (doxorubicin, DOX), and an NIR dye (IR780). ${ }^{24}$ Since the melting point of the eutectic mixture was slightly higher than the physiological temperature of human bodies, the encapsulated DOX remained unreleased when incubated with cancer cells at $37{ }^{\circ} \mathrm{C}$. As shown in Fig. 5B, after cellular uptake, DOX was basically confined in endolysosomes, which was characterized by a clear punctate pattern (for the group designated as $0 \mathrm{~min}$ ). Upon NIR irradiation, DOX started to escape from endolysosomes and subsequently accumulated in the nucleus, which was indicated by the blurred fluorescence in the cytosol as well as the emergence of the nuclear outline, indicating the feasibility of NIR irradiation for the regulation of drug release from NSFA nanoparticles. In addition to NIR light, other external stimuli are also promising to trigger on-demand drug release from NSFA-based materials, such as ultrasound-triggered hyperthermia and alternating magnetic field-induced heat generation. ${ }^{102-104}$

\subsection{Targeted drug delivery}

Active targeting represents an efficient strategy for guiding payloads to be delivered to specific types of cells and/or microenvironments by equipping the carriers with targeting ligands to facilitate strong ligand-receptor interactions (e.g., the high affinity between the arginine-glycine-aspartic acid (RGD) motif and $\alpha_{\mathrm{v}} \beta_{3}$ integrin). ${ }^{105-107}$ In contrast to passive targeting, active targeting largely improves the accumulation of payloads in the diseased site and thus reduces undesired side effects on healthy tissues. It is demonstrated that NSFA-based materials could be decorated with targeting ligands (e.g., peptides) for active targeting. ${ }^{108}$ In spite of these achievements, native biological materials with intrinsic tumor-targeting capability are still preferred due to their biocompatibility and biodegradability as well as non-immunogenicity. In an example, Zhu et al.
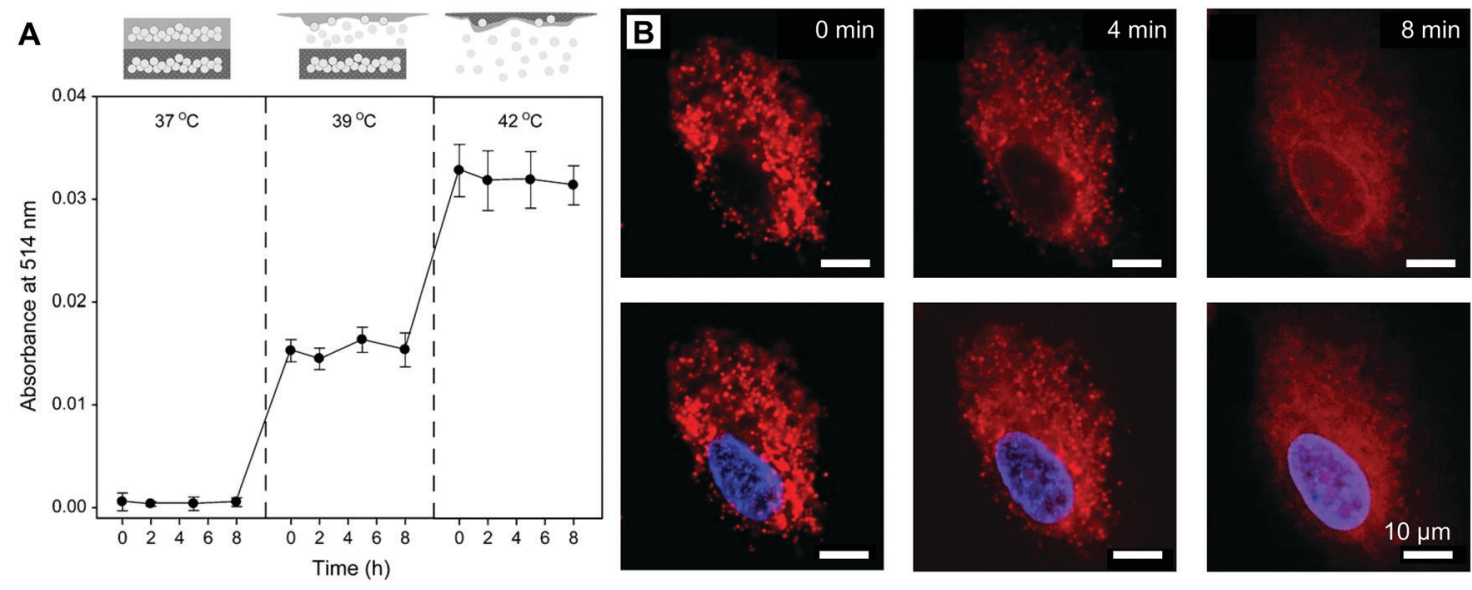

Fig. 5 Controlled drug release. (A) The release profile of FITC-dextran from gelatin microbeads embedded in a dual PCM block fabricated from 1-tetradecanol and LA. Reproduced from ref. 23 with permission from Wiley- $\mathrm{VCH}$. (B) Confocal laser scanning microscopy (CLSM) images of DOX release from an NIR-responsive, phospholipid-stabilized NSFA nanoparticle after uptake by A549 cells upon irradiation with an $808 \mathrm{~nm}$ laser. DOX is shown in red. The nucleus was stained by Hoechst 33342 and is shown in blue. Reproduced from ref. 24 with permission from Wiley-VCH. 
reconstituted the hydrophobic core of a native nanocarrier LDL with a eutectic mixture of LA and SA and DOX using a solventextraction strategy as discussed earlier. ${ }^{67}$ By means of the specific binding of ApoB-100 toward LDL receptors, the reconstituted LDL particles were actively ingested by LDL receptor-overexpressing A549 cancer cells, whereas no remarkable uptake was found in normal NIH-3T3 fibroblast cells. Furthermore, both targeted killing of cancer cells in vitro and selective imaging of tumor tissues in vivo were achieved.

In a recent study, our group further extended the application of such a system by replacing the anticancer drug with a mitochondrion-targeting, aggregation-induced emission (AIE) photosensitizer (Fig. 6A). ${ }^{68}$ In particular, the AIE photosensitizer possessed typical AIE and twisted intramolecular charge transfer (TICT) characteristics as well as reactive oxygen species (ROS)-sensitizing capability, enabling the application of the resultant reconstituted $\mathrm{LDL}$ (i.e., $\operatorname{rLDL}(+)$ particles) for fluorescence-feedback PDT. ${ }^{109-111}$ As shown in Fig. 6B and C, the receptor-blocking experiment with a 20 -fold excess of native LDL demonstrated that the reconstitution process did not compromise the targeting capability of ApoB-100 toward A549 cells. Once the $\operatorname{rLDL}(+)$ particles were ingested by A549 cells and subsequently digested for releasing the mitochondriontargeting AIE photosensitizer, the cells were subjected to in situ light irradiation. The bright-field images shown in Fig. 6D indicated that there was a significant change in the cellular morphology due to the generation of considerable ROS, including cell blebbing and chromatin condensation, which were the characteristic features of cell apoptosis. Interestingly, this process could be monitored in a real-time fashion, as reflected by the remarkably enhanced luminescence and blue-shifted emission due to the AIE and TICT characteristics of the photosensitizer (Fig. 6E), which was presumably attributed to the increased mechanical stress during apoptosis. Quantitative cell viability analysis suggested that the photodynamic killing efficiency of rLDL(+) particles toward A549 cells was up to $c a$. 88\%, indicating the outstanding PDT effect of such a system.

\subsection{Cancer therapy}

Cancer is a collection of diseases involving uncontrolled growth of abnormal cells with the potential to invade or spread to other parts of the body, which poses severe threats to human health. ${ }^{112}$ To fight against cancer, a number of research directions have been established, in which cancer nanomedicine has emerged as the most promising branch for the treatment of cancer. ${ }^{113}$ By integration with various theranostic agents, NSFAbased systems have demonstrated their potential for cancer therapy, including the incorporation of anti-cancer drugs (e.g., doxorubicin, paclitaxel, and camptothecin) for chemotherapy, photothermal agents (e.g., ICG, IR780, and gold nanocages)
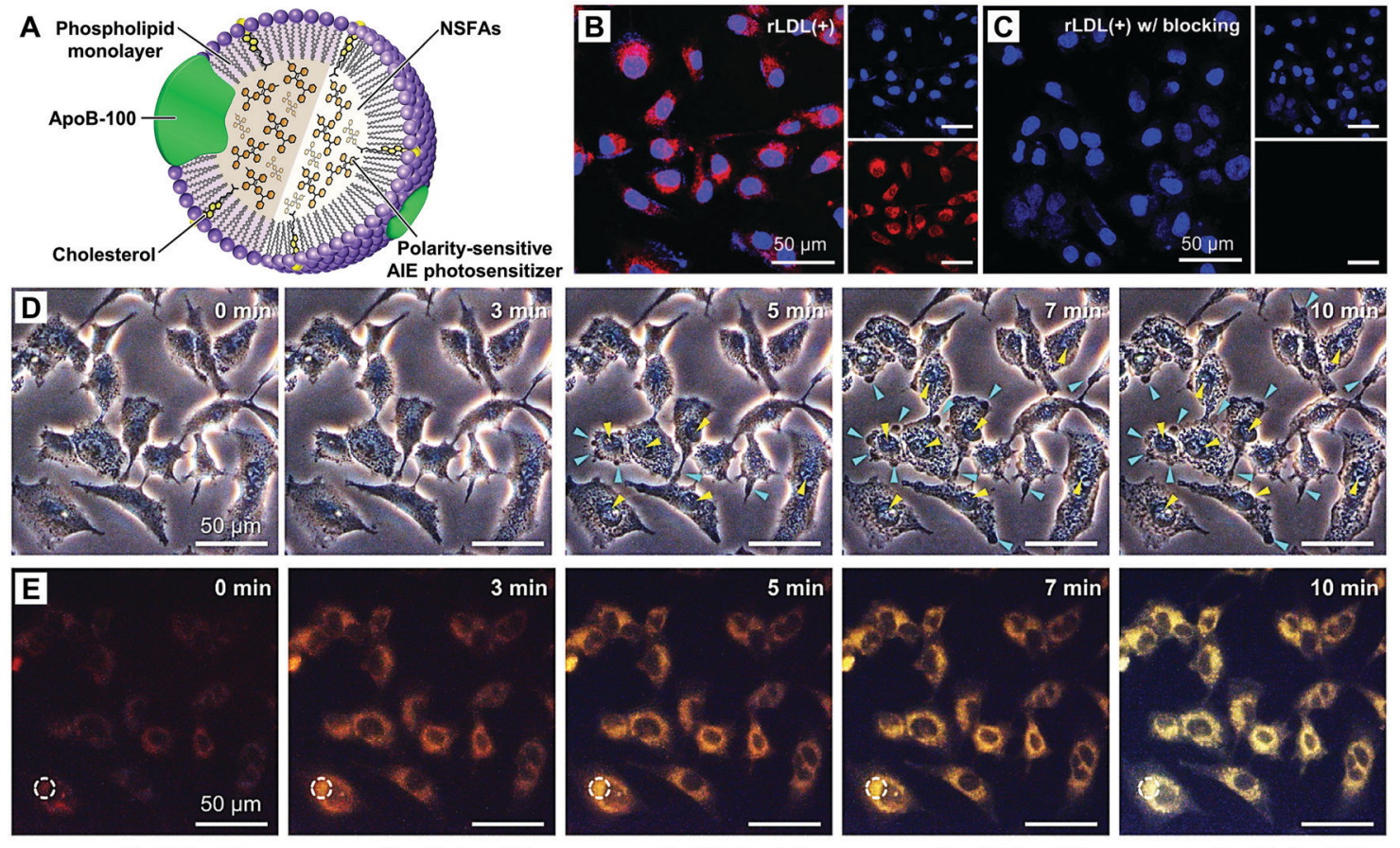

Fig. 6 NSFA-reconstituted LDL particles for fluorescence-feedback PDT. (A) A schematic LDL particle reconstituted with NSFAs and a polarity-sensitive AIE photosensitizer (i.e., $r L D L(+)$ particles). (B) Cellular uptake of $r L D L(+)$ particles. (C) Cellular uptake of rLDL(+) particles in the presence of a 20 -fold excess of native LDLs. The AIE photosensitizer is shown in red. The nucleus was stained by Hoechst 33342 and is shown in blue. (D) Optical micrographs showing PDT-induced cell apoptosis. The blue and yellow arrows indicate cell blebbing and chromatin condensation, respectively. (E) Fluorescence images showing fluoresence-feedback PDT. The fluoresence signals in all images originated from the photosensitizer, in which the colored bars indicate the hue $(\mathrm{H})$ and lightness $(\mathrm{L})$ values (from 0 to 255 ) taken from the dashed circle using PowerPoint 2019. Reproduced from ref. 68 with permission from the Royal Society of Chemistry. 
for photothermal therapy (PTT), and photosensitizers (e.g., porphyrin and other functional molecules) for PDT. ${ }^{24,55,79,88,114,115}$ In addition, a plethora of imaging contrast agents (e.g., fluorescent dyes, photoacoustic agents, and magnetic resonance contrast agents) have been employed to build nanotheranostic platforms for the visualization of tumor tissues and facilitate the integration of multiple functionalities for precision cancer medicine. ${ }^{55,116}$ In a study, Fan and coworkers reported an NSFAbased all-in-one multifunctional nanoplatform, which was composed of a eutectic core of LA and SA, DOX, a multifunctional small molecule (DPP-BT), and a folic-acid functionalized phospholipid shell. ${ }^{55}$ In this system, DPP-BT served as the contrast agent for NIR-II fluorescence and photoacoustic (PA) imaging as well as the therapeutic agent for PTT and PDT. Upon NIR irradiation, DOX was efficiently released from the NSFA-based nanoparticles via photothermal heating by DPPBT. Furthermore, both NIR-II fluorescence/PA dual-modal imaging and combined PTT/PDT/chemotherapy have been demonstrated on tumor-bearing mice simply by irradiation with a single NIR laser, indicating the advantage of this all-inone nanoplatform.

Since most NIR-responsive NSFA-based systems do not have selectivity toward the complicated tumor microenvironment, it is highly desired to construct a smart system that is specifically activated at the tumor site. To this end, Zhao and coworkers developed an NSFA-based activatable nanoplatform for imaging-guided cancer therapy, in which a clinical anticancer drug camptothecin-11 (CPT-11) and an $\mathrm{H}_{2} \mathrm{~S}$-activated NIR photothermal agent (InTBOD-Cl) were co-loaded into a eutectic mixture of LA and SA. ${ }^{114}$ In particular, the presence of $\mathrm{H}_{2} \mathrm{~S}$, an upregulated biomarker in various types of cancer, significantly converted the NIR-transparent InTBOD-Cl into a new molecule InTBOD-SH that had strong absorption in the NIR-I region and sufficient emission in the NIR-II region (Fig. 7A). Upon irradiating the resultant nanoparticles (NP@BOD/CPT) with an NIR laser, the photothermal agent generated in situ by $\mathrm{H}_{2} \mathrm{~S}$ rapidly elevated the system temperature, resulting in the melting of NSFAs and subsequent release of CPT-11 (Fig. 7B). Notably, in the absence of $\mathrm{H}_{2} \mathrm{~S}$, no evident photothermal conversion could be induced, limiting the off-target leakage of CPT-11. Furthermore, both in vitro cell viability assay in $\mathrm{H}_{2} \mathrm{~S}$-rich human colorectal cancer HCT116 cells and in vivo NIR-II imaging-guided anticancer experiments in HCT116 tumor-bearing mice were performed to demonstrate the effectiveness of such a system (Fig. 7C), holding great promise in precision medicine with minimal side effects on healthy tissues.
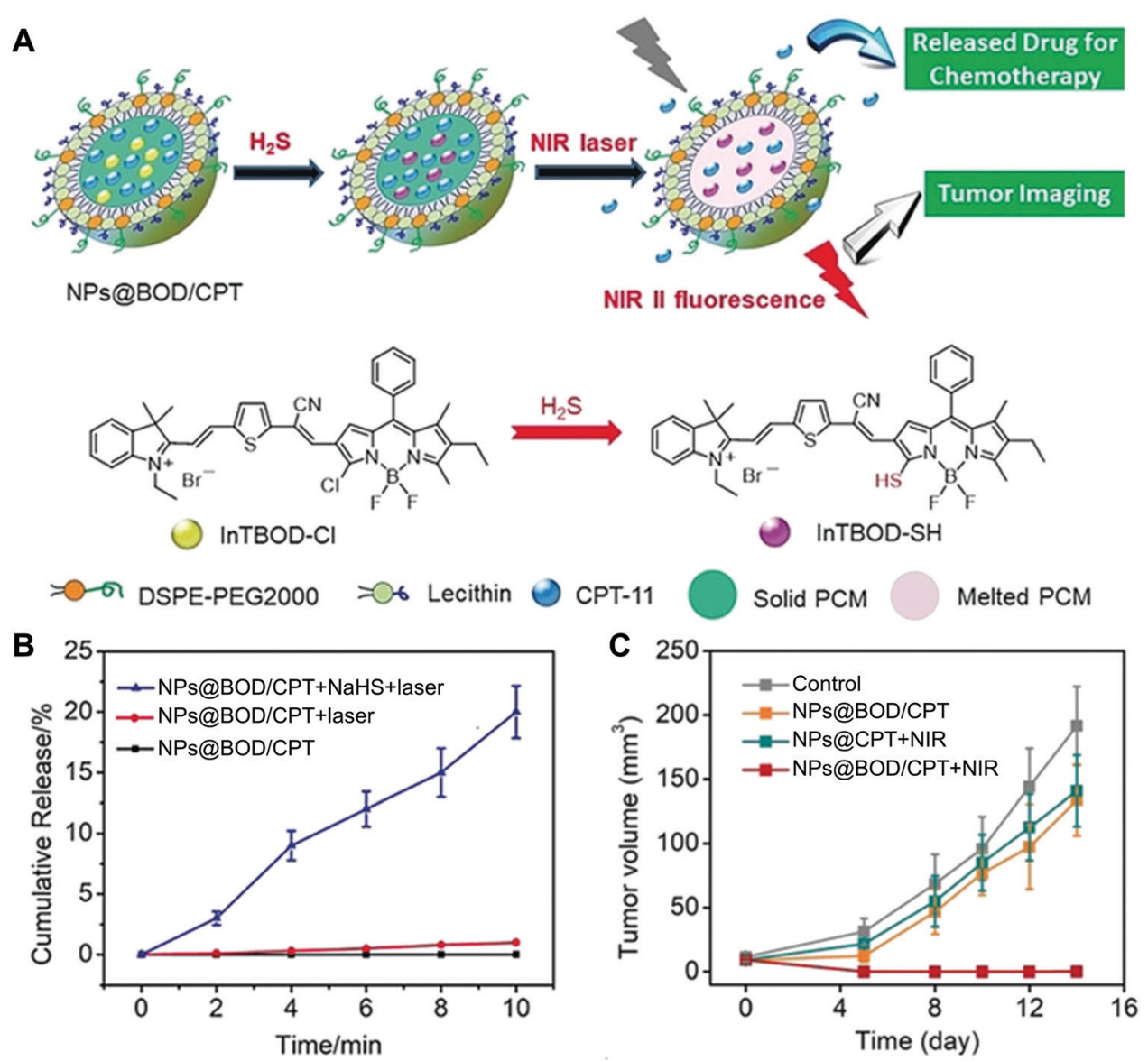

Fig. 7 NSFA-based activatable nanoparticles for imaging-guided cancer therapy. (A) Schematic illustration showing $\mathrm{H}_{2} \mathrm{~S}$-mediated in situ generation of photothermal transducers for cancer imaging and on-demand drug release. (B) Cumulative release profiles of CPT-11 from NP@BOD/CPT after various treatments. (C) Tumor growth curves of HCT116 tumor-bearing mice in various groups. Reproduced from ref. 114 with permission from Wiley-VCH. 
Despite the fact that the intake of appropriate amounts of NSFAs is not detrimental to human health, excessive ingestion of NSFAs is considered to be harmful, which increases the risk of cardiovascular or liver diseases. ${ }^{117-119}$ Interestingly, recent studies showed that certain NSFAs have selective inhibition effects on malignant cells. For example, Lappano et al. evidenced that LA was able to promote apoptosis of breast and endometrial cancer cells via inducing ROS generation, stimulating the phosphorylation of epidermal growth factor receptor (EGFR), and altering gene expression. ${ }^{120}$ Reczyńska et al. suggested that a large intake of NSFAs by cancerous A549 cells led to decreased stiffness of cell membranes and reduced cell viability, whereas the cell viability of non-malignant BEAS-2B cells was barely affected. ${ }^{121}$ Such a discrepancy indicates that the delivery of excessive NSFAs to cancer cells may provide an alternative way for targeted cancer therapy.

\subsection{Antibacterial treatment}

The development of biocompatible materials for antibacterial therapy has attracted extensive attention. ${ }^{122-124}$ Aside from the aforementioned physiochemical properties, NSFAs also possess intrinsic antibacterial activity, which opens the door for using NSFAs as bactericides for efficient killing of pathogenic bacteria. ${ }^{30,31,125}$ Despite the fact that the exact antibacterial mechanisms of NSFAs remain unclear, related studies suggest that the primary targets of NSFAs lie in the bacterial cell membrane (membrane destabilization by acting as detergentlike behaviors) and membrane-located processes (e.g., disruption of electron transport chains and uncoupling of oxidative phosphorylation). ${ }^{126,127}$ In addition, the secondary degradation products of NSFAs (e.g., peroxidation, which generates $\mathrm{H}_{2} \mathrm{O}_{2}$ and other ROS) may also contribute to bacterial growth inhibition. $^{128}$

Taking advantage of the intrinsic antibacterial activity of NSFAs, our group recently developed an exceptional broadspectrum NSFA-based nanobiocide for multimodal and synergistic inactivation of drug-resistant bacteria. ${ }^{58}$ It was found that a copper-LA complex could be formed via physical blending of excess LA and cupric acetate followed by efficient ligand exchange. Interestingly, the resulting composite materials not only exhibited prominent photothermal effects but also extended the antibacterial spectrum of LA from Gram-positive bacteria to both Gram-positive and Gram-negative bacteria. By adopting the aforementioned in situ surface polymerization technique, the composite materials were transformed into colloidally stable nanoparticles. In particular, the surface of the resulting nanoparticles was intentionally decorated with net positive charges via the introduction of positively charged monomers to facilitate efficient binding with negatively charged bacteria. Combining the LA-mediated membrane-damaging effect, a copper-mediated Fenton-like reaction, and the photothermal effect of the copper-LA complex, a broad-spectrum, multimodal, and synergistic antibacterial effect was achieved both in vitro and in vivo. Surprisingly, the killing efficiency was determined to be $99.99 \%$ for ampicillin-resistant Escherichia coli $\left(\mathrm{Amp}^{\mathrm{r}}\right.$ E. coli) and 99.9999\% for methicillin-resistant
Staphylococcus aureus (MRSA), indicating the potential of such a system for the treatment of drug-resistant infections in clinical settings.

By virtue of the thermo-responsive properties of NSFAs, Liang and coworkers reported an NSFA-based, phospholipidstabilized triple-function nanoparticle (i.e., TRIDENT) for efficient chemo-photothermal therapy of multidrug-resistant bacterial infection. ${ }^{56}$ In this system, a eutectic mixture of LA and SA was used as the gating material, while imipenem (IMP) and IR780 served as antibiotic and photothermal/fluorescent agents, respectively (Fig. 8A). Upon NIR irradiation, IR780 molecules generated heat to irreversibly damage the structure of bacteria. Meanwhile, the melted NSFAs led to the efficient release of IMP for chemical inactivation of bacterial growth. In vitro studies suggested that the irradiation of TRIDENT with an NIR laser (i.e., the group denoted as IMP/IR780@TRN + NIR) gave rise to efficient inhibition against both multidrug-resistant E. coli and MRSA, with basically no colonies appearing on agar plates (Fig. 8B). In contrast, all other control groups did not show such an obvious antibacterial effect, indicating the advantage of this synergistic antibacterial system. Furthermore, the antibacterial behaviors of TRIDENT were investigated on animal models, in which mouse skin was pre-infected with multidrug-resistant $E$. coli and MRSA, respectively. As shown in Fig. $8 \mathrm{C}$ and $\mathrm{D}$, the overall recovery situation of the infected areas was in accordance with the bactericidal effect in vitro, with the group of IMP/IR780@TRN + NIR achieving almost full recovery in both infection models on day 15 . These results validated that the NSFA-based multifunctional nanoparticles could effectively kill multidrug-resistant bacteria at a low antibiotic dose and thus reduce the adverse effects associated with the administration of high-dose drugs. Based on a similar NSFA-based nanosystem with the payloads replaced by calcium peroxide $\left(\mathrm{CaO}_{2}\right)$ and rifampicin (RFP, an antibiotic), Han and coworkers established an endogenous stimuli-powered nanoreactor for combination therapy of bacterial infections. ${ }^{129}$ Intrinsically, pathogenic bacteria (e.g., MRSA) are able to produce a wide range of virulence factors, in which bacterial toxins are the most sophisticated one. In particular, the resulting nanoreactors could efficiently capture $\alpha$-toxin secreted by MRSA in vivo, leading to the formation of pores on the surface of the nanoreactors and thus the penetration of water to react with $\mathrm{CaO}_{2}$ and produce hydrogen peroxide $\left(\mathrm{H}_{2} \mathrm{O}_{2}\right)$. Subsequently, the decomposition of $\mathrm{H}_{2} \mathrm{O}_{2}$ to oxygen accelerated the release of RFP. In addition, the nanoreactors with captured toxins could further stimulate the body's immune responses, which significantly alleviated the virulence of toxins and enhanced the therapeutic effect on bacterial infections.

\subsection{Tissue engineering}

Tissue engineering uses a combination of scaffold design, cellular control, and biological signaling to promote regeneration of injured tissues. ${ }^{130-132}$ Among various elements, growth factors play a crucial role in tissue regeneration to facilitate cell proliferation, differentiation, migration, adhesion, and gene expression. Notable examples of growth factors include insulin 
A
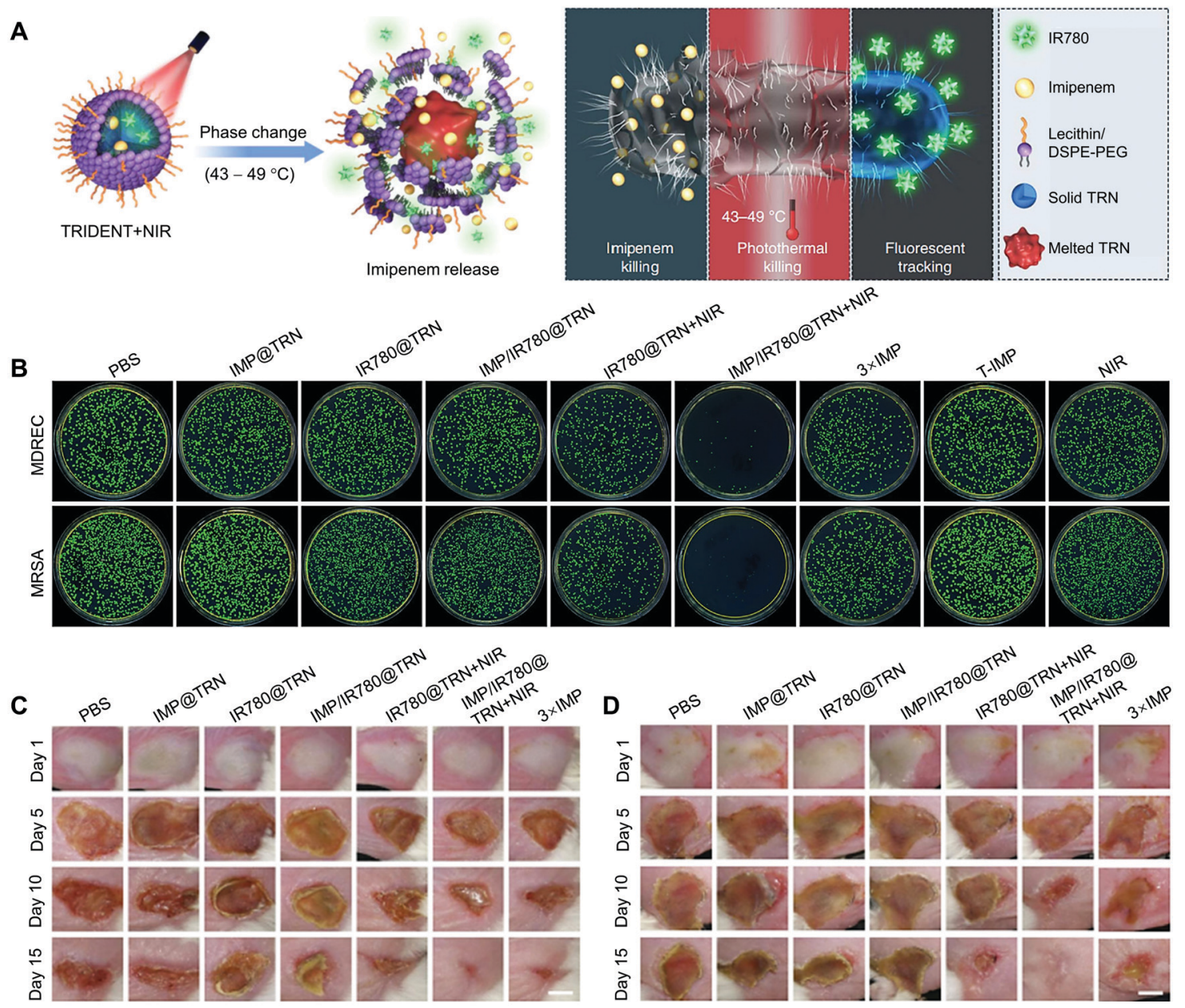

Fig. 8 NSFA-based tri-function nanoparticles for efficient chemo-photothermal therapy of multidrug-resistant bacterial infection. (A) Schematic illustration showing the working mechanism of TRIDENT. (B) In vitro antibacterial activities of TRIDENT against clinically multidrug-resistant bacteria (multidrug-resistant E. coli and MRSA). (C and D) Photographs of (C) multidrug-resistant E. coli-infected skin and (D) MRSA-infected skin of mice during treatment with different formulations for 15 days. Reproduced from ref. 56 with permission from Springer Nature.

growth factor (IGF), fibroblast growth factor (FGF), transforming growth factor beta (TGF- $\beta$ ), and nerve growth factor (NGF). ${ }^{133}$ In general, growth factors exhibit short-range diffusion in the extracellular matrix and act locally due to the short half-lives and slow diffusion, which is not conducive to tissue development and regeneration. ${ }^{134}$ These issues can be partially addressed by encapsulating growth factors into responsive materials for on-demand release. Xia and coworkers explored such an application by fabricating NSFA-based microparticles using coaxial electrospray, in which the outer and inner solutions contained NSFAs and payloads (i.e., NGF and an NIR dye ICG), respectively. ${ }^{135}$ Furthermore, their application in neural tissue engineering was demonstrated. Specifically, a tri-layered construct was obtained by sandwiching the resulting microparticles ( $5.6 \pm 1.8 \mu \mathrm{m}$ in size) between two layers of electrospun fibers, in which one layer was comprised of random fibers to provide mechanical strength, and the other layer was made of uniaxially aligned fibers to offer physical guidance for neurite outgrowth. Upon NIR irradiation, ICG elevated the system temperature to melt the NSFA matrix, leading to the prompt release of biologically active NGF. Under these circumstances, the neurite outgrowth of spheroids of PC12 cells (a classical neuronal cell model) on the tri-layered construct was significantly promoted. By varying the growth factors and/or the scaffolding materials, this on-demand release system can also be applied to other types of tissue engineering.

Related studies suggest that medium-chain NSFAs (e.g., CA and LA) are capable of augmenting NGF-induced neurite outgrowth from PC12 cells via the activation of mitogenactivated protein kinases. ${ }^{136}$ Motivated by this cognition, Xia and co-workers fabricated solid NSFA microparticles using electrospray and further deposited them on uniaxially aligned electrospun microfibers with varying density for investigating the outgrowth of neurites. ${ }^{137}$ The NSFA microparticles $(4.36 \pm$ $0.33 \mu \mathrm{m}$ in size) were composed of a eutectic mixture of LA and SA with a melting point of $39{ }^{\circ} \mathrm{C}$, which enabled the structural intactness of the resulting microparticle when incubated with cells at $37{ }^{\circ} \mathrm{C}$. Uniaxially aligned poly-(E-caprolactone) (PCL) microfibers fabricated by electrospinning were utilized to collect NSFA microparticles for different periods of time, generating 

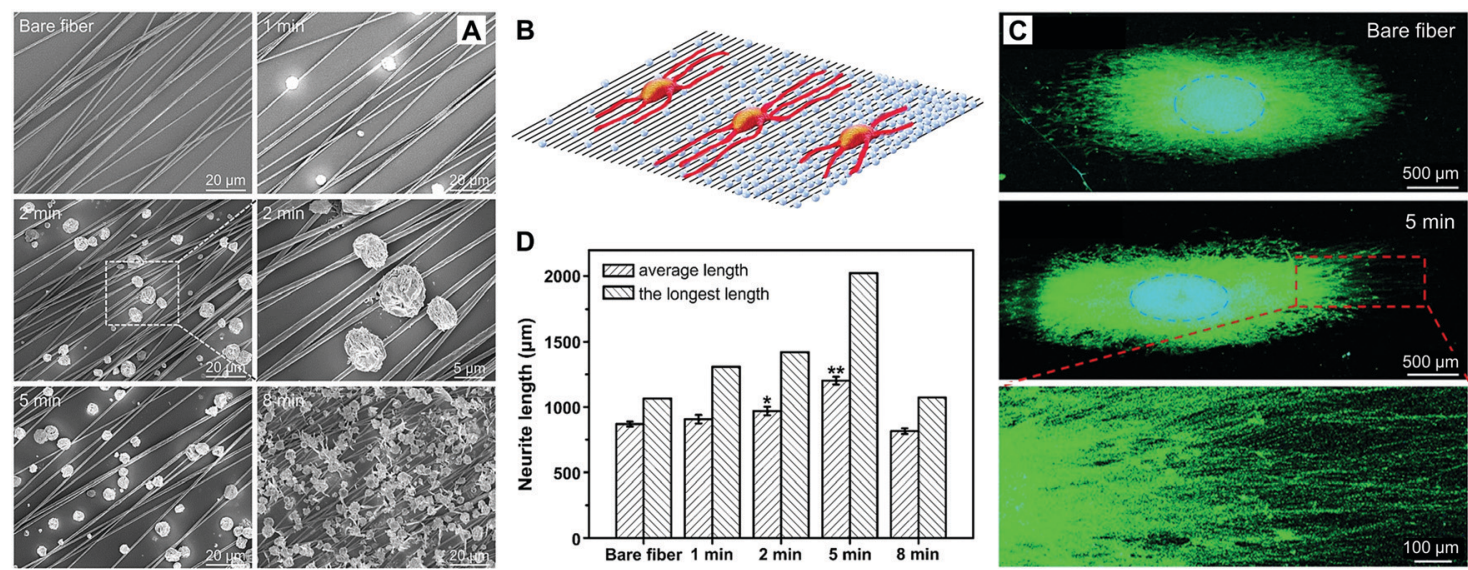

Fig. 9 NSFA-based electrosprayed microparticles for promoting the outgrowth of neurites on electrospun microfibers. (A) SEM images of electrosprayed microparticles collected on uniaxially aligned, electrospun PCL fibers for 0, 1, 2, 5, and 8 min, respectively. (B) Schematic illustration showing the outgrowth of neurites on uniaxially aligned electrospun microfibers. (C) Fluorescence images of typical neurites extending from chick embryonic DRG cultured on uniaxially aligned fibers deposited with NSFA microparticles for 0 and $5 \mathrm{~min}$. The neurites were immunostained with Tuj1 marker (green), and the nuclei were stained with 4',6-diamidino-2-phenylindole (blue). (D) The average and the longest lengths of the neurites extending from DRG cultured on uniaxially aligned fibers deposited with NSFA microparticles for $0,1,2,5$, and 8 min, respectively, which were measured from the corresponding fluorescence images. ${ }^{*} p<0.05$ and ${ }^{* *} p<0.01$. Reproduced from ref. 137 with permission from Wiley-VCH.

PCL microfibers with a varying coating density of NSFA microparticles (Fig. 9A). Furthermore, chick embryonic dorsal root ganglia (DRG) bodies were employed as a neuron model to examine the directional outgrowth of neurites on these substrates (Fig. 9B). As shown in Fig. 9C, the neurites extending from the DRG cultured on the substrates were in accordance with the orientation of the aligned fibers. Quantitative analysis indicated that, in contrast to bare fibers, the fibers deposited with microparticles for 5 min gave rise to a remarkably increased average length (from $869.6 \pm 19.1 \mu \mathrm{m}$ to $1202.3 \pm 29.7 \mu \mathrm{m}$ ) and the longest length of the neurites (Fig. 9D). However, further increasing the coating density (collection time $=8 \mathrm{~min}$ ) would cause cytotoxicity to the cells and thus inhibit the outgrowth of neurites. This study suggests that the neurite outgrowth can be enhanced by a synergistic effect of the chemical cue (i.e., NSFAs), the surface roughness arising from the microparticles, and the topographical cue provided by the aligned PCL microfibers.

\section{Summary and outlook}

As a class of promising biomaterials, NSFAs have attracted extensive attention due to their unique properties in terms of low cost, chemical stability, well-defined melting points, large heat of fusion, reversible solid-liquid phase transition, biocompatibility, biodegradability, and inherent antibacterial activity. In view of these advantages, a plethora of attempts have been made by taking NSFAs as gating materials for controlled release or simply serving as bioactive substances for the manipulation of bacterial/cellular behaviors, which greatly boosts their widespread applications in biomedical research. In this review, we systematically summarized the advances of NSFAbased materials in the biomedical field over the past decade. We first introduced the concept of NSFAs and their physiochemical/biological properties, with an emphasis on the working mechanism for controlled release. We then discussed the stabilization strategies for the fabrication of colloidally dispersed NSFA particles. Furthermore, we showcased the specific applications of NSFA-based materials in biomedical research, including controlled drug release, targeted drug delivery, cancer therapy, antibacterial treatment, and tissue engineering.

Despite these exciting achievements, there is still huge room to further the development of NSFA-based materials in the biomedical field. (i) Integration with other bio-based PCMs. Naturally occurring fatty alcohols (e.g., dodecanol, tetradecanol, and hexadecanol) are another type of bio-based PCMs with similar physiochemical properties to NSFAs, which can also be used as thermo-responsive materials for controlled release. $^{22,138}$ For example, Dong and coworkers reported a smart nanoplatform for multi-mode imaging-guided synergistic cancer therapy, in which the encapsulation matrix was composed of a eutectic mixture of stearyl alcohol and linoleic acid (m. p. $=43.9^{\circ} \mathrm{C}$ ) for an NIR-triggered phase transition. ${ }^{138}$ Interestingly, the use of stearyl alcohol and linoleic acid greatly reduced skin phototoxicity during PDT due to their protective effect, holding great potential for safe phototherapy. In contrast to NSFAs, the crystallization capability of fatty alcohols is relatively poor, which provides a possible solution to decrease the crystallinity of NSFAs through co-mixing with other PCMs for facile formulation and efficient loading of exogenous substances. ${ }^{139}$ In addition, fatty alcohols are also capable of forming eutectic mixtures with NSFAs, which can further extend the available melting points of NSFAs. (ii) Exact biological behaviors of NSFAs in organisms. Although NSFAs have the capacity to inhibit or kill bacteria and cancer cells (with an excessive intake), there is still a lack of direct evidence to interpret the exact mechanism. Besides, free NSFAs are able to stimulate mammalian cells to produce lipid droplets; 
however, the precise mechanism of lipid droplet formation remains elusive. ${ }^{140-142}$ Systematic investigation of these unclear biological processes is conducive to promote the establishment of NSFA-based targeted theranostic systems. (iii) Temperature control. As an outstanding class of PCMs, NSFAs have found widespread applications in energy-storage applications owing to their large heat of fusion. During phase transitions, NSFAs store or release large heat energy with the temperature keeping constant. Through rational experimental design and the selection of appropriate photothermal agents, it is possible to achieve constant temperature control (preferably within the mild hyperthermia range), ensuring safe PTT without sacrificing the surrounding healthy tissues. (iv) Temperature sensing. NSFAs can respond to temperature variations by exhibiting a reversible solid-liquid phase transition, which is accompanied by a series of physical properties, such as crystallinity and viscosity. By integration with appropriate chemical or biological sensors that are able to exhibit signal changes to these physical parameters, it is promising to achieve sensitive temperature sensing. To sum up, the biomedical use of NSFAs is still in its infant stage, which will surely play increasingly important roles through in-depth exploration by researchers from diverse disciplines. It is hoped that this review can motivate a number of ideas and further promote the development of NSFA-based systems for biomedical use.

\section{Conflicts of interest}

There are no conflicts to declare.

\section{Acknowledgements}

This work was financially supported by the National Natural Science Foundation of China (52003123) and Start-up Funds from Nankai University (to C. Z.).

\section{Notes and references}

1 C. M. Scrimgeour and J. L. Harwood, Fatty Acid and Lipid Structure, The Lipid Handbook, Harwood, 2007.

2 F. D. Gunstone, Fatty Acid and Lipid Chemistry, Blackie Academic \& Professional, Glasgow, 1996.

3 C. Scrimgeour, Bailey's Industrial Oil and Fat Products, American Cancer Society, Hoboken, 2005.

4 S. W. White, J. Zheng, Y. M. Zhang and C. O. Rock, Annu. Rev. Biochem., 2005, 74, 791-831.

5 C. C. C. R. de Carvalho and M. J. Caramujo, Molecules, 2018, 23, 2583.

6 N. Turner, G. J. Cooney, E. W. Kraegen and C. R. Bruce, J. Endocrinol., 2014, 220, T61-T79.

7 E. J. Steen, Y. Kang, G. Bokinsky, Z. Hu, A. Schirmer, A. McClure, S. B. Del Cardayre and J. D. Keasling, Nature, 2010, 463, 559-562.

8 H. Maag, J. Am. Oil Chem. Soc., 1984, 61, 259-267.
9 I. Johansson and M. Svensson, Curr. Opin. Colloid Interface Sci., 2001, 6, 178-188.

10 L. J. Stolp, P. J. Gronlund and D. R. Kodali, J. Am. Oil Chem. Soc., 2019, 96, 727-738.

11 Y. Yuan, N. Zhang, W. Tao, X. Cao and Y. He, Renewable Sustainable Energy Rev., 2014, 29, 482-498.

12 D. Rozanna, T. G. Chuah, A. Salmiah, T. S. Y. Choong and M. Sa'ari, Int. J. Green Energy, 2005, 1, 495-513.

13 D. C. Hyun, N. S. Levinson, U. Jeong and Y. Xia, Angew. Chem., Int. Ed., 2014, 53, 3780-3795.

14 B. Zalba, B. J. M. Marin, L. F. Cabeza and H. Mehling, Appl. Therm. Eng., 2003, 23, 251-283.

15 A. Sharma, V. V. Tyagi, C. R. Chen and D. Buddhi, Renewable Sustainable Energy Rev., 2009, 13, 318-345.

16 L. F. Cabeza, J. Illa, J. Roca, F. Badia, H. Mehling, S. Hiebler and F. Ziegler, Mater. Corros., 2001, 52, 140-146.

17 R. Kumar, M. K. Misra, R. Kumar, D. Gupta, P. K. Sharma, B. B. Tak and S. R. Meena, Def. Sci. J., 2011, 61, 576-582.

18 D. Sharma, H. Kitano and K. Sagara, Res. Rep. Fac. Eng., Mie Univ., 2004, 29, 31-64.

19 S. Hasnain, Energy Convers. Manage., 1998, 39, 1127-1138.

20 J. Daniel and R. Rajasekharan, J. Am. Oil Chem. Soc., 2003, 80, 417-421.

21 H. M. Schaink, K. F. van Malssen, S. Morgado-Alves, D. Kalnin and E. van der Linden, Food Res. Int., 2007, 40, 1185-1193.

22 J. Qiu, D. Huo and Y. Xia, Adv. Mater., 2020, 32, 2000660.

23 S.-W. Choi, Y. Zhang and Y. Xia, Angew. Chem., Int. Ed., 2010, 49, 7904-7908.

24 C. Zhu, D. Huo, Q. Chen, J. Xue, S. Shen and Y. Xia, Adv. Mater., 2017, 29, 1703702.

25 A. C. Rustan and C. A. Drevon, Encyclopedia of Life Sciences, 2005.

26 S. M. Sadrameli, W. Seames and M. Mann, Fuel, 2008, 87, 1776-1780.

27 E. Currie, A. Schulze, R. Zechner, T. C. Walther and R. V. Farese, Cell Metab., 2013, 18, 153-161.

28 D. G. Mashek and R. A. Coleman, Curr. Opin. Lipidol., 2006, 17, 274-278.

29 A. Carracedo, L. C. Cantley and P. P. Pandolfi, Nat. Rev. Cancer, 2013, 13, 227-232.

30 A. P. Desbois and V. J. Smith, Appl. Microbiol. Biotechnol., 2010, 85, 1629-1642.

31 J. B. Parsons, J. Yao, M. W. Frank, P. Jackson and C. O. Rock, J. Bacteriol., 2012, 194, 5294-5304.

32 P. C. Calder, J. Parenter. Enteral Nutr., 2015, 39, 18S-32S.

33 D. Ackerman, S. Tumanov, B. Qiu, E. Michalopoulou, M. Spata, A. Azzam, H. Xie, M. C. Simon and J. J. Kamphorst, Cell Rep., 2018, 24, 2596-2605.

34 S. Phan, S. Salentinig, E. Gilbert, T. A. Darwish, A. Hawley, R. Nixon-Luke, G. Bryant and B. J. Boyd, J. Colloid Interface Sci., 2015, 449, 160-166.

35 W. Mehnert and K. Mäder, Adv. Drug Delivery Rev., 2012, 64, 83-101.

36 G. D. Moon, S. W. Choi, X. Cai, W. Li, E. C. Cho, U. Jeong, L. V. Wang and Y. Xia, J. Am. Chem. Soc., 2011, 133, 4762-4765. 
37 S. Xie, L. Zhu, Z. Dong, Y. Wang, X. Wang and W. Zhou, Int. J. Nanomed., 2011, 6, 547-555.

38 S. Kumar and J. K. Randhawa, RSC Adv., 2015, 5, 68743-68750.

39 A. Beloqui, M. A. Solinís, A. Rodríguez-Gascón, A. J. Almeida and V. Préat, Nanomedicine, 2016, 12, 143-161.

40 Y. Yuan, W. Tao, X. Cao and L. Bai, J. Chem. Eng. Data, 2011, 56, 2889-2891.

41 M. Li, Z. Wu and H. Kao, Sol. Energy Mater. Sol. Cells, 2011, 95, 2412-2416.

42 L. Wang and D. Meng, Appl. Energy, 2010, 87, 2660-2665. 43 A. Karaipekli and A. Sarı, J. Sci. Ind. Res., 2007, 66, 470-476. 44 A. Sarı, H. Sarı and A. Önal, Energy Convers. Manage., 2004, 45, 365-376.

45 A. Sarı, Appl. Therm. Eng., 2005, 25, 2100-2107.

46 Y. Cai, H. Ke, L. Lin, X. Fei, Q. Wei, L. Song, Y. Hu and H. Fong, Energy Convers. Manage., 2012, 64, 245-255.

47 A. Karaipekli and A. Sarı, J. Ind. Eng. Chem., 2010, 16, 767-773.

48 A. Karaipekli and A. Sarı, Renewable Energy, 2008, 33, 2599-2605.

49 P. Zhao, Q. Yue, H. He, B. Gao, Y. Wang and Q. Li, Appl. Energy, 2014, 115, 483-490.

50 W. Aftab, X. Huang, W. Wu, Z. Liang, A. Mahmood and R. Zou, Energy Environ. Sci., 2018, 11, 1392-1424.

51 C. Liu, Z. Rao, J. Zhao, Y. Huo and Y. Li, Nano Energy, 2015, 13, 814-826.

52 M. S. Nikolic, C. Olsson, A. Salcher, A. Kornowski, A. Rank, R. Schubert, A. Fromsdorf, H. Weller and S. Forster, Angew. Chem., Int. Ed., 2009, 48, 2752-2754.

53 L. Zhang, J. M. Chan, F. X. Gu, J.-W. Rhee, A. Z. Wang, A. F. Radovic-Moreno, F. Alexis, R. Langer and O. C. Farokhzad, ACS Nano, 2008, 2, 1696-1702.

54 K. Letchford and H. Burt, Eur. J. Pharm. Biopharm., 2007, 65, 259-269.

55 Q. Wang, Y. Dai, J. Xu, J. Cai, X. Niu, L. Zhang, R. Chen, Q. Shen, W. Huang and Q. Fan, Adv. Funct. Mater., 2019, 29, 1901480.

56 G. Qing, X. Zhao, N. Gong, J. Chen, X. Li, Y. Gan, Y. Wang, Z. Zhang, Y. Zhang, W. Guo, Y. Luo and X.-J. Liang, Nat. Commun., 2019, 10, 4336.

57 Q. Chen, C. Zhu, D. Huo, J. Xue, H. Cheng, B. Guan and Y. Xia, Nanoscale, 2018, 10, 22312-22318.

58 K. Xue, C. Yang, C. Wang, Y. Liu, J. Liu, L. Shi and C. Zhu, CCS Chem., 2021, 3, 531-544.

59 M. Yan, J. Du, Z. Gu, M. Liang, Y. Hu, W. Zhang, S. Priceman, L. Wu, Z. H. Zhou, Z. Liu, T. Segura, Y. Tang and Y. Lu, Nat. Nanotechnol., 2010, 5, 48-53.

60 L. Han, C. Liu, H. Qi, J. Zhou, J. Wen, D. Wu, D. Xu, M. Qin, J. Ren, Q. Wang, L. Long, Y. Liu, I. Chen, X. Yuan, Y. Lu and C. Kang, Adv. Mater., 2019, 31, 1805697.

61 J. Cao, Y. Zhao, Y. Liu, S. Tian, C. Zheng, C. Liu, Y. Zhai, Y. An, H. J. Busscher, L. Shi and Y. Liu, ACS Macro Lett., 2019, 8, 651-657.

62 E. V. Batrakova and M. S. Kim, J. Controlled Release, 2015, 219, 396-405.
63 C. S. Thaxton, J. S. Rink, P. C. Naha and D. P. Cormode, Adv. Drug Delivery Rev., 2016, 106, 116-131.

64 J.-W. Yoo, D. J. Irvine, D. E. Discher and S. Mitragotri, Nat. Rev. Drug Discovery, 2011, 10, 521-535.

65 K. K. Ng, J. F. Lovell and G. Zheng, Acc. Chem. Res., 2011, 44, 1105-1113.

66 C. Zhu and Y. Xia, Chem. Soc. Rev., 2017, 46, 7668-7682.

67 C. Zhu, P. Pradhan, D. Huo, J. Xue, S. Shen, K. Roy and Y. Xia, Angew. Chem., Int. Ed., 2017, 56, 10399-10402.

68 C. Wang, X. Zhao, H. Jiang, J. Wang, W. Zhong, K. Xue and C. Zhu, Nanoscale, 2021, 13, 1195-1205.

69 B. Xu, Q. Cao, Y. Zhang, W. Yu, J. Zhu, D. Liu and G. Jiang, ACS Biomater. Sci. Eng., 2018, 4, 2473-2483.

70 Y. Li, J. Lu, J. Zhang, X. Zhu, J. Liu and Y. Zhang, ACS Appl. Bio Mater., 2020, 3, 8705-8713.

71 C. Ji, W. Cheng, Y. Hu, Y. Liu, F. Liu and M. Yin, Nano Today, 2021, 36, 101020.

72 J. Xu, Y. Zhou, H. Nie, Z. Xiong, H. OuYang, L. Huang, H. Fang, H. Jiang, F. Huang, Y. Yang, X. Ding, X. Wang and W. Zhou, J. Mater. Chem. B, 2020, 8, 787-793.

73 C. Liu, Y. Cao, Y. Cheng, D. Wang, T. Xu, L. Su, X. Zhang and H. Dong, Nat. Commun., 2020, 11, 1735.

74 F. Han, R. Wang, Y. Feng, S. Wang, L. Liu, X. Li, Y. Han and H. Chen, Nat. Commun., 2019, 10, 1548.

75 G. Guan, Z. Zhang, Z. Wang, B. Liu, D. Gao and C. Xie, Adv. Mater., 2007, 19, 2370-2374.

76 S. Hyuk Im, U. Jeong and Y. Xia, Nat. Mater., 2005, 4, 671-675.

$77 \mathrm{~J}$. Qiu, J. Xu and Y. Xia, Adv. Healthcare Mater., 2021, 10, 2000587.

78 D. C. Hyun, P. Lu, S.-I. Choi, U. Jeong and Y. Xia, Angew. Chem., Int. Ed., 2013, 52, 10468-10471.

79 J. Qiu, D. Huo, J. Xue, G. Zhu, H. Liu and Y. Xia, Angew. Chem., Int. Ed., 2019, 58, 10606-10611.

80 Y. Luan, M. Yang, Q. Ma, Y. Qi, H. Gao, Z. Wu and G. Wang, J. Mater. Chem. A, 2016, 4, 7641-7649.

81 X. Li, Y. Liu, F. Fu, M. Cheng, Y. Liu, L. Yu, W. Wang, Y. Wan and Z. Yuan, Nano-Micro Lett., 2019, 11, 68.

82 S. E. Skrabalak, L. Au, X. Li and Y. Xia, Nat. Protoc., 2007, 2, 2182-2190.

83 S. E. Skrabalak, J. Chen, Y. Sun, X. Lu, L. Au, C. M. Cobley and Y. Xia, Acc. Chem. Res., 2008, 41, 1587-1595.

84 J. Chen, M. Yang, Q. Zhang, E. C. Cho, C. M. Cobley, C. Kim, C. Glaus, L. V. Wang, M. J. Welch and Y. Xia, $A d v$. Funct. Mater., 2010, 20, 3684-3694.

85 J. Chen, B. Wiley, Z.-Y. Li, D. Campbell, F. Saeki, H. Cang, L. Au, J. Lee, X. Li and Y. Xia, Adv. Mater., 2005, 17, 2255-2261.

86 M. S. Yavuz, Y. Cheng, J. Chen, C. M. Cobley, Q. Zhang, M. Rycenga, J. Xie, C. Kim, K. H. Song, A. G. Schwartz, L. V. Wang and Y. Xia, Nat. Mater., 2009, 8, 935-939.

87 H. Cheng, D. Huo, C. Zhu, S. Shen, W. Wang, H. Li, Z. Zhu and Y. Xia, Biomaterials, 2018, 178, 517-526.

88 S. Shen, C. Zhu, D. Huo, M. Yang, J. Xue and Y. Xia, Angew. Chem., Int. Ed., 2017, 56, 8801-8804.

89 Q. Chen, D. Huo, H. Cheng, Z. Lyu, C. Zhu, B. Guan and Y. Xia, Adv. Healthcare Mater., 2018, 8, 1801113. 
90 S. Mura, J. Nicolas and P. Couvreur, Nat. Mater., 2013, 12, 991-1003.

91 A. P. Blum, J. K. Kammeyer, A. M. Rush, C. E. Callmann, M. E. Hahn and N. C. Gianneschi, J. Am. Chem. Soc., 2015, 137, 2140-2154.

92 Y. Wang, A. Santos, A. Evdokiou and D. Losic, J. Mater. Chem. B, 2015, 3, 7153-7172.

93 Y. Wang, S. Song, J. Liu, D. Liu and H. Zhang, Angew. Chem., Int. Ed., 2015, 54, 536-540.

94 N. Lee, D. Yoo, D. Ling, M. H. Cho, T. Hyeon and J. Cheon, Chem. Rev., 2015, 115, 10637-10689.

95 S. Gao, G. Tang, D. Hua, R. Xiong, J. Han, S. Jiang, Q. Zhang and C. Huang, J. Mater. Chem. B, 2019, 7, 709-729.

96 C. V. Harper, D. J. Woodcock, C. Lam, M. Garcia-Albornoz, A. Adamson, L. Ashall, W. Rowe, P. Downton, L. Schmidt, S. West, D. G. Spiller, D. A. Rand and M. R. H. White, Proc. Natl. Acad. Sci. U. S. A., 2018, 115, E5243-E5249.

97 D. Fukumura and R. K. Jain, J. Cell. Biochem., 2007, 101, 937-949.

98 X. Yang, M. Yang, B. Pang, M. Vara and Y. Xia, Chem. Rev., 2015, 115, 10410-10488.

99 Q. Xin, H. Shah, A. Nawaz, W. Xie, M. Z. Akram, A. Batool, L. Tian, S. U. Jan, R. Boddula, B. Guo, Q. Liu and J. R. Gong, Adv. Mater., 2018, 31, 1804838.

100 J. Huang, B. He, Z. Zhang, Y. Li, M. Kang, Y. Wang, K. Li, D. Wang and B. Z. Tang, Adv. Mater., 2020, 32, 202003382.

101 N. Lee and T. Hyeon, Chem. Soc. Rev., 2012, 41, 2575-2589.

102 J. Fu, T. Li, Y. Zhu and Y. Hao, Adv. Funct. Mater., 2019, 29, 1906195.

103 Q. Zhang, J. Liu, K. Yuan, Z. Zhang, X. Zhang and X. Fang, Nanotechnology, 2017, 28, 405101.

104 G. Huang, Y. Qiu, F. Yang, J. Xie, X. Chen, L. Wang and H. Yang, Nano Lett., 2021, 21, 2926-2931.

105 Z. Zhao, A. Ukidve, J. Kim and S. Mitragotri, Cell, 2020, 181, 151-167.

106 M. T. Manzari, Y. Shamay, H. Kiguchi, N. Rosen, M. Scaltriti and D. A. Heller, Nat. Rev. Mater., 2021, 6, 351-370.

107 R. Pasqualini, E. Koivunen and E. Ruoslahti, Nat. Biotechnol., 1997, 15, 542-546.

108 Z. Gao, T. Shi, Y. Li, C. You, K. Cheng and B. Sun, Colloids Surf., B, 2019, 176, 230-238.

109 J. Mei, N. L. C. Leung, R. T. K. Kwok, J. W. Y. Lam and B. Z. Tang, Chem. Rev., 2015, 115, 11718-11940.

110 C. Zhu, R. T. K. Kwok, J. W. Y. Lam and B. Z. Tang, ACS Appl. Bio Mater., 2018, 1, 1768-1786.

111 S. Liu, X. Zhou, H. Zhang, H. Ou, J. W. Y. Lam, Y. Liu, L. Shi, D. Ding and B. Z. Tang, J. Am. Chem. Soc., 2019, 141, 5359-5368.

112 Y. Dai, C. Xu, X. Sun and X. Chen, Chem. Soc. Rev., 2017, 46, 3830-3852.

113 C. Cao, N. Yang, H. Dai, H. Huang, X. Song, Q. Zhang and X. Dong, Nanoscale Adv., 2021, 3, 106-122.

114 B. Shi, N. Ren, L. Gu, G. Xu, R. Wang, T. Zhu, Y. Zhu, C. Fan, C. Zhao and H. Tian, Angew. Chem., Int. Ed., 2019, 58, 16826-16830.
115 T.-K. Ryu, S.-W. Baek, R.-H. Kang, K.-Y. Jeong, D.-R. Jun and S.-W. Choi, J. Controlled Release, 2018, 270, 237-245.

116 Y. Dai, J. Su, K. Wu, W. Ma, B. Wang, M. Li, P. Sun, Q. Shen, Q. Wang and Q. Fan, ACS Appl. Mater. Interfaces, 2019, 11, 10540-10553.

117 M. A. Briggs, K. S. Petersen and P. M. Kris-Etherton, Healthcare, 2017, 5, 29.

118 R. Micha and D. Mozaffarian, Lipids, 2010, 45, 893-905.

119 D. Wang, Y. Wei and M. J. Pagliassotti, Endocrinology, 2006, 147, 943-951.

120 R. Lappano, A. Sebastiani, F. Cirillo, D. C. Rigiracciolo, G. R. Galli, R. Curcio, R. Malaguarnera, A. Belfiore, A. R. Cappello and M. Maggiolini, Cell Death Discovery, 2017, 3, 17063.

121 K. Reczyńska, D. Khanal, K. Pielichowska, E. Pamuła and W. Chrzanowski, Lipids, 2020, 55, 117-126.

122 B. Joseph, V. K. Sagarika, C. Sabu, N. Kalarikkal and S. Thomas, J. Bioresour. Bioprod., 2020, 5, 223-237.

123 H. Huang, L. Mao, Z. Li, Y. Liu, S. Fan, Y. Jin and J. Xie, J. Bioresour. Bioprod., 2019, 4, 231-241.

124 H. Xu, D. Zhang and J. Li, J. Bioresour. Bioprod., 2019, 4, 177-182.

125 J. M. Silva, E. Silva, R. L. Reis and A. R. C. Duarte, Sustainable Chem. Pharm., 2019, 14, 100192.

126 Y. M. Zhang and C. O. Rock, Nat. Rev. Microbiol., 2008, 6, 222-233.

127 C. W. Sheu and E. Freese, J. Bacteriol., 1972, 111, 516-524.

128 P. Schönfeld and L. Wojtczak, Free Radical Biol. Med., 2008, 45, 231-241.

129 Y. Wu, Z. Song, H. Wang and H. Han, Nat. Commun., 2019, 10, 4464.

130 B. P. Chan and K. W. Leong, Eur. Spine J., 2008, 17, 467-479.

131 F. Rey, B. Barzaghini, A. Nardini, M. Bordoni, G. V. Zuccotti, C. Cereda, M. T. Raimondi and S. Carelli, Cells, 2020, 9, 1636.

132 F. J. O'Brien, Mater. Today, 2011, 14, 88-95.

133 P. Tayalia and D. J. Mooney, Adv. Mater., 2009, 21, 3269-3285.

134 K. Lee, E. A. Silva and D. J. Mooney, J. R. Soc., Interface, 2011, 8, 153-170.

135 J. Xue, C. Zhu, J. Li, H. Li and Y. Xia, Adv. Funct. Mater., 2018, 28, 1705563.

136 Y. Kamata, H. Shiraga, A. Tai, Y. Kawamoto and E. Gohda, Neuroscience, 2007, 146, 1073-1081.

137 J. Xue, T. Wu, J. Li, C. Zhu and Y. Xia, Angew. Chem., Int. Ed., 2019, 58, 3948-3951.

138 D. Chen, Y. Tang, J. Zhu, J. Zhang, X. Song, W. Wang, J. Shao, W. Huang, P. Chen and X. Dong, Biomaterials, 2019, 221, 119422.

139 H. B. Ghadim, K. Shahbaz, R. Al-Shannaq and M. M. Farid, Int. J. Energy Res., 2019, 43, 8536-8547.

140 J. A. Olzmann and P. Carvalho, Nat. Rev. Mol. Cell Biol., 2019, 20, 137-155.

141 A. L. S. Cruz, E. d. A. Barreto, N. P. B. Fazolini, J. P. B. Viola and P. T. Bozza, Cell Death Dis., 2020, 11, 105.

142 P. Shyu, X. F. A. Wong, K. Crasta and G. Thibault, Biosci. Rep., 2018, 38, BSR20180764. 